\title{
Impact of Small Cells Overlapping on Mobility Management
}

\author{
Ali Mahbas, Huiling Zhu, Senior Member, IEEE, and Jiangzhou Wang, Fellow, IEEE
}

\begin{abstract}
The mobility management will be more complex and will have a great impact on the quality of service $(\mathrm{QoS})$ in the future cellular networks, as these networks will have to handle a huge number of user equipment (UEs) and their frequent handoffs due to very dense short-footage small cells. This paper presents a framework to model and derive the coverage of small cells, the cell sojourn time and the handoff rate in multi-tier small cell networks. The distribution of the small cells around a reference UE's path is studied by taking into consideration the overlaps among the small cells. Two types of handoff rates are introduced to estimate the load managed by different cells, where inter-frequency handoff (IRH) rate and intra-frequency handoff (IAH) rate represent the fraction of handoffs managed by the first tier and the other tiers, respectively. Our analysis shows that ignoring the overlaps among the small cells affects the accuracy of the results significantly. The simulation results validate the accuracy of the analytical results and also show the impact of different parameters such as the small cell density, the number of tiers and the size of the small cells on the small cell sojourn time, the macro cell sojourn time and the handoff rate.
\end{abstract}

Index Terms-Mobility management, small cell networks, cell sojourn time, handoff rate, small cells coverage.

\section{INTRODUCTION}

Similar to the resource management [1], [2], the mobility management is important and essential in the cellular systems [3]. It is anticipated that the number of user equipment (UEs) will increase rapidly and small cells with different frequency (e.g. high frequency) will be deployed densely in the next generation of cellular systems. The mobility management in these systems will be very challenging, and developing an accurate model to evaluate the system performance is essential. Both the handoff rate and the cell sojourn time are used not only for network dimensioning and estimating the signalling overload, but also for estimating the UEs' speed [4]-[6]. Furthermore, finding the time that the UEs spend in the small cells coverage can help to estimate the power consumption in the small cell discovery process [7]-[9].

In the dense multi-tier heterogeneous networks (HetNets), taking the overlaps among the small cells into account when modeling the high frequency small cells coverage is essential for accurate speed estimation, estimating the energy consumption in the small cell discovery, and estimating the required resources at the different cells. For instance, estimating the

A. Mahbas was with the School of Engineering and Digital Arts, University of Kent, Canterbury, CT2 7NT, United Kingdom. He is now with the Iraqi Ministry of Communication (MOC), Baghdad, Iraq (e-mail: ajm83@kentforlife.net)

H. Zhu and J. Wang are with the School of Engineering and Digital Arts, University of Kent, Canterbury, CT2 7NT, United Kingdom (e-mail: H.Zhu@kent.ac.uk; J.Z.Wang@kent.ac.uk) time that UEs spend in the first tier and, the number of handoffs that the first tier is involved in, will help in estimating the required resources (e.g. signaling and frequency) at the overloaded macro cells. Ignoring the overlaps among the small cells will result in misleading information. In recent years, the handoff rate and the cell sojourn time in the cellular systems have received significant attention [4], [5], [10]-[12]. The handoff rate and the cell sojourn time in a single-tier network were studied in [11]. However, the future cellular network will include multiple tiers with different frequency bands (e.g. high frequency small cells), and in the presence of small cells, the mobility management is more complex and more system parameters need to be considered.

When studying the mobility management in the HetNets, modelling the cells has taken two main directions, the Voronoi Tessellations cells (VTCs) assumption and the regular shapes assumption (e.g. circle and hexagonal). Regarding the first direction, in the conventional HetNets (all tiers use the same frequency channel) different tiers in the network are assumed to form VTCs. Stochastic geometry was used in [13] to propose a framework for vertical and horizontal handoff rates experienced by a UE with arbitrary movement trajectory in cochannel HetNets. Although VTCs assumption is reasonable for this type of deployment, it is expected that the future HetNets will include dense small cells operating on different frequency channels. The VTCs assumption has also been considered in the inter-frequency HetNet [4], [5]. In [4], the number of handoffs made during a time window was used to estimate the UE's speed in dense small cell networks. Stochastic geometry was used to derive approximations to the Cramer-Rao lower bound (CRLB) for the speed estimate of a UE. In [5], the UE's speed was estimated by using the sojourn time, where CRLB for the sojourn time-based speed estimation was analysed. Both papers [4], [5] assumed that the single-tier small cell in the network forms VTCs which means that the whole network is covered by the small cells. However, a huge infrastructure will be required for the high frequency small cells to cover the whole network, as the high frequency suffers from very large propagation loss [14]. Also this assumption restricts the analysis to a one-tier cellular system similar to [11].

Considering the second direction, both papers [10] and [12] assumed that the small cells in two-tier HetNets have regular shapes. In [10], the cell sojourn time in a two-tier HetNet was addressed where the small cells were assumed to have fixed hexagonal shapes in the network and the overlap coverage among the small cells was not taken into consideration. In [12], it is investigated the mobility in a two-tier HetNet and also derived the sojourn time and the cross-tier handoff rate. The 
overlapping among small cells of ellipse shapes on a reference UE's path was also neglected in this work. Therefore, some of the intra-tier handoffs (handoffs among small cells due to overlapping) will be counted as cross-tier handoffs. Ignoring the overlaps will not only affect the accuracy of the handoff rate but also affect the accuracy of the cell sojourn time as shown later in this paper.

The randomness in the network deployment, which causes more complexity in the analysis, is considered as one of the main challenges that the recent studies have dealt with. Therefore, some assumptions were made in these studies in order to minimize this complexity and obtain insights regarding the system resources utilization and UE's quality of service (QoS). It is expected that these assumptions affect the accuracy of the analysis in dense HetNets, which may cause misleading information. The main objective in this paper is to propose a novel framework to study the main mobility management parameters while taking into consideration the trend of deploying small cells densely in the system. As a result, the distances among these small cells are very short which causes overlapping. As will be shown in this paper, these overlaps affect both the handoff rate and the cell sojourn time of the macro and the small cells significantly. The future HetNets will include small cells with different sizes to cover different areas, such as shops and shopping centers. Although considering multiple tiers of small cells increases the complexity of the analysis, unlike other studies [4], [5], [7], [9], [10], [12] which considered single-tier small cells, this paper considers multi-tier small cells in order to provide insights regarding the mobility management in more realistic scenarios.

The contribution of this paper is summarized as follows:

- The locations of the small cell base stations (SCBSs), the macro cell base stations (MCBSs) and the waypoints of a reference UE during its movement in the system are randomly distributed on the plane and form independent Poisson point processes (PPPs). The distribution of SCSBs around a reference UE's path is studied and the small cells crossed by the reference UE during one movement is mapped into marked point process (MPP) on $\mathbb{R}^{+}$. This assumption is validated through simulations.

- Based on the above mapping, a novel framework is proposed to model the coverage of a one-tier small cell network and the overlap coverage among these small cells on the reference UE's path. The proposed framework is expanded to a multi-tier small cell framework where each tier is characterized with a different transmit power, pathloss exponent, and density.

- Two types of handoffs are also introduced in this paper, an inter-frequency handoff (IRH) and an intra-frequency handoff (IAH). The IRH is defined as the handoff taking place between cells that use different frequency channels, e.g. between the high frequency small cells and the macro cells, and the IAH is defined as the handoff taking place between cells that use the same frequency, e.g. high frequency small cells. Both the IRH rate and the IAH rate can help to estimate the amount of signalling needed for handoffs at both the overloaded macro cells (first tier) and the high frequency small cells.

- This paper further addresses the cell sojourn time in a multi-tier HetNet where the expectation of the cell sojourn time is derived by using the proposed framework. Results are presented to show the impact of different system parameters such as the density of the small cells, the transmit power of different small cell tiers and the different mobility characteristics, on the availability of small cells, the handoff rates and the cell sojourn time.

The rest of this paper is structured as follows: Section II describes the system model and the mobility model. The small cells coverage on a reference UE's path is investigated in Section III. In Section IV, the small cell sojourn time and the macro cell sojourn time in a multi-tier HetNet are derived. In Section V, the total handoff, the IAH and the IRH rates are studied and derived. In Section VI, the system performance is shown by numerical and simulation results. Conclusions are drawn in Section VII.

\section{SySTEM MODEL}

The future HetNet will adopt the inter-frequency deployment where the large cells (macro cells) use low frequency to provide the coverage and, due to the limitation of low frequency bandwidths, the smaller cells (small cells) use high frequency (this is because high frequencies are available with wide bandwidths). The small cells will be deployed densely in the network at hot spots and users' premises for data rate and capacity enhancement. Furthermore, the small cells may have different footprints (or different sizes) to cover different areas, such as shops and shopping centers. In this paper, we consider a $K$-tier HetNet in Fig. 1 , where $K \geq 1$. When $K=1$, only macro cells are considered in the system, while three tiers of small cells next to the macro cells are considered when $K=4$. Note that the tier refers to a set of cells that transmit with the same power and use the same frequency channel. Each tier is characterized with the tuple $\left\{p_{k}, \lambda_{k}, \alpha_{k}\right\}$, where $p_{k}$ is the transmit power, $\lambda_{k}$ is the density of the base stations and $\alpha_{k}$ is the path-loss exponent of the $k$ th tier. It is assumed that the first-tier uses a low frequency and a high frequency is reused at each small cell from tiers $2,3, \cdots, K$. For tractability and clarifying the system model, we make the following assumptions:

- PPP Assumption: It is assumed that MCBSs and SCBSs in the network are randomly distributed as independent PPP $\Phi_{k}$ with density $\lambda_{k}$, where $k \in[1,2, \cdots, K][15]$.

- Minimum Received Power Association (MRPA): The load imbalance and minimization of the small cells' coverage may take place in the inter-frequency HetNets due to big differences in the transmit powers and the propagation losses [14], [16]. Since there is no interference between the first tier and the other tiers, it is assumed that the association to the small cells is based on the minimum received power $\left(\rho_{\text {min }}\right)$ from any small cell. Furthermore, the association among the tiers $2,3, \cdots, K$ is based on the maximum average received power. Any UE will be 
associated to the $j$ th small cell of the $m$ th tier when the received power satisfies the condition below:

$$
\rho_{\min } \leq \rho_{m, j} \geq \max _{i \in \Phi_{n}} \rho_{n, i}
$$

where $m, n \in[2,3, \cdots, K], \rho_{\min }$ is the minimum received power to consider the UEs in the small cells' coverage, $\rho_{m, j}$ and $\rho_{n, i}$ are the received power from the $j$ th small cell of the $m$ th tier and the $i$ th small cell of the $n$th tier respectively.

- Open-Access Assumption (OAA): It is also assumed that all small cells operate in open-access mode. The UEs in the system can associate to any small cell as long as the requirements of the association scheme mentioned in MRPA are met.

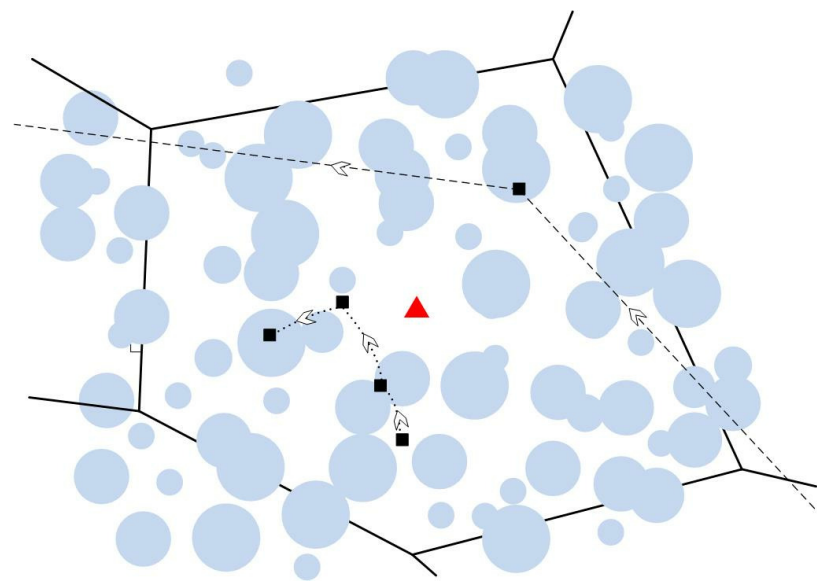

Fig. 1: System Model. Red triangles represent the locations of the MCBSs, blue dots represent the small cells coverage (different small cell tiers), black squares represent the waypoints, and the black dashed and dotted lines represent the reference UE's path between any two waypoints for long and short transition lengths respectively.

\section{A. Association}

When the location is chosen randomly in the system, Stochastic Geometry is used to derive the small cell association. From MRPA, the probability that a reference UE $\left(\mathcal{U}_{0}\right)$ is associated to any small cell can be obtained as follows.

Lemma 1 The probability of $\mathcal{U}_{0}$ associated to any small cell in the system is expressed as:

$$
\mathcal{A}_{\bar{K}}=1-\exp \left(-\pi \sum_{m=2}^{K} \lambda_{k}\left(\frac{\rho_{\text {min }}}{\mathscr{L}_{2} p_{m}}\right)^{\frac{-2}{\alpha_{m}}}\right)
$$

where $\bar{K}$ represents the set of the small cell tiers (it also represents the number of the small cell tiers $\bar{K}=K-1$ ). $\mathscr{L}_{2}$ is the path-loss of the high frequency at 1 meter.

Proof: Without loss of generality, we assume that $\mathcal{U}_{0}$ is located at the origin and $\mathcal{A}_{k}$ is the probability that $\mathcal{U}_{0}$ is associated to the $k$ th tier. Thus the probability of $\mathcal{U}_{0}$ associated to the first tier is given by:

$$
\begin{aligned}
\mathcal{A}_{1} & =\prod_{m=2}^{K} \mathbb{P}\left[\rho_{m, 0}<\rho_{\text {min }}\right] \\
& =\prod_{m=2}^{K} \mathbb{P}\left[R_{m, 0}>\left(\frac{\rho_{m i n}}{\mathscr{L}_{2} p_{m}}\right)^{\frac{-1}{\alpha_{m}}}\right] \\
& =\prod_{m=2}^{K} \exp \left(-\pi \lambda_{m}\left(\frac{\rho_{m i n}}{\mathscr{L}_{2} p_{m}}\right)^{\frac{-2}{\alpha_{m}}}\right)
\end{aligned}
$$

where $\mathbb{P}[$.$] indicates the probability, \rho_{m, 0}$ is the received power from the nearest small cell of the $m$ th tier, $R_{m, 0}$ is the distance to the nearest SCBS of the $m$ th tier, and $\exp \left(-\pi \lambda_{m}\left(\frac{\rho_{m i n}}{\mathscr{L}_{2} p_{m}}\right)^{\frac{-2}{\alpha_{m}}}\right)$ is the probability of no SCBS of the $m$ th tier within the area $\pi R_{m, 0}^{2}$ and it is obtained from the null probability [15]. Eq. (2) is obtained from $\mathcal{A}_{\bar{K}}=\mathcal{A}_{K}-\mathcal{A}_{1}$ where $\mathcal{A}_{K}=\sum_{k=1}^{K} \mathcal{A}_{k}=1$.

Note that the result in Lemma 1 is used in the analysis to obtain other parameters such as the expected pause time in different tiers and the cell sojourn time.

When $\mathcal{U}_{0}$ moves, it is associated to different cells according to MRPA. For instance, when $\mathcal{U}_{0}$ is associated to the first tier, the handoff is initiated from the serving macro cell to the small cell with strongest signal strength if $\mathcal{U}_{0}$ receives a signal strength of a value $\rho_{\min }$ or greater from any small cell. The serving macro cell triggers the inter-frequency scan at $\mathcal{U}_{0}$ periodically to establish whether $\mathcal{U}_{0}$ receives $\rho_{\text {min }}$ from any surrounding small cell or not [8]. The handoff from the small cells to the first tier will take place when $\mathcal{U}_{0}$ leaves the coverage of its serving small cell and the received signal strength from the small cell with strongest signal is less than $\rho_{\text {min }}$. Furthermore, the handoff between two small cells occurs when $\mathcal{U}_{0}$ moves between two overlapping small cells. The handoff procedure will be initiated to a neighbouring small cell when the received signal from serving small cell becomes less than the signal from the neighbouring small cell. The time that $\mathcal{U}_{0}$ is associated to the small cells will be derived later.

\section{B. Mobility Model}

The classical random waypoint (RWP) model suffers from several issues [11] [17] [18]. The first issue is that the stationary spatial node distribution tends to concentrate on the center of the finite domain when UEs are uniformly distributed in the network. The second issue concerns the transition lengths in the classical RWP. These transition lengths are the same order as the size of the domain. In [11], a new RWP was proposed to mitigate some of the issues that the classic RWP suffers from. In the proposed RWP model, the direction of the movement is chosen uniformly in $[0,2 \pi]$ and each of the transition, the speed and pause time are chosen from some distribution. The RWP proposed in [11] is considered in this paper. The movement trace of a UE is modeled by the quadruples $\left\{W_{l-1}, W_{l}, V_{l}, S_{l}\right\}_{l \in \mathbb{L}}$ where $l$ denotes the $l$ th movement. During the $l$ th movement, $W_{l-1}$ and $W_{l}$ denote the starting waypoint and destination waypoint, respectively, and $V_{l}$ and $S_{l}$ denote the velocity of the UE and pause time respectively. The velocities $V_{l}$ are independent and identically distributed (i.i.d.) with distribution $P_{V}($.$) and$ pause times $S_{l}$ are i.i.d with distribution $P_{S}($.$) . The waypoints$ $\left\{W_{0}, W_{1}, \cdots W_{l-1}, W_{l}, \cdots W_{L}\right\}$ are a homogeneous PPP $\Phi_{w}(l)$ with density $\lambda_{w}$, and the nearest point in $\Phi_{w}(l)$ is selected as the destination waypoint:

$$
W_{l}=\underset{w \in \Phi_{w}(l)}{\arg \min }\left\|w-W_{l-1}\right\|
$$

where $\|$. $\|$ indicates the Euclidean distance. The transition lengths $\left\{\left\|W_{1}-W_{0}\right\|,\left\|W_{2}-W_{1}\right\| \cdots\left\|W_{l}-W_{l-1}\right\| \cdots\right\}$ are 
i.i.d. Therefore, for brevity we consider $\mathcal{U}_{0}$ 's path from $W_{0}$ to $W_{1}\left(\mathcal{P}_{0}\right)$. The expected transition length is obtained as:

$$
\mathbb{E}\left[\left\|W_{1}-W_{0}\right\|\right]=\frac{1}{2 \sqrt{\lambda_{w}}}
$$

The mean transition time can be obtained similar to [11] as:

$$
\mathbb{E}\left[T_{0}\right]= \begin{cases}\frac{1}{2 v \sqrt{\lambda_{w}}}, & V=v \\ \frac{\ln v_{\max }-\ln v_{\min }}{2 \sqrt{\lambda_{w}}\left(v_{\max }-v_{\min }\right)}, & V \sim N\left(v_{\min }, v_{\max }\right)\end{cases}
$$

\section{Multi-Tier Small Cells}

In this section, we investigate the coverage of the small cells on $\mathcal{P}_{0}$ by taking into consideration that some overlap may take place on the path. Since each tier of small cells has a different footprint, the $j$ th small cell of the $m$ th tier is crossed by $\mathcal{U}_{0}$, if its BS is located at a distance of $r_{m, j}$ from the $\mathcal{P}_{0}$, where $r_{j, m}$ is radius of the $j$ th small cell's coverage. When $D_{m, j}$ represents the vertical distance between $\mathcal{P}_{0}$ and the $j$ th SCBS from the $m$ th tier, the number of small cells crossed by $\mathcal{U}_{0}$ is:

$$
N_{0}=\sum_{m=2}^{K} \sum_{j \in \Phi_{m}} \mathbf{1}\left(D_{m, j} \leq r_{m, j}\right)
$$

where $\mathbf{1}($.$) is the indicator function. Since the small cells from$ the $m$ th tier are randomly distributed in the system as PPP, the number of small cells from the $m$ th tier in a bounded set $\mathscr{A}$ has a Poisson distribution with mean $\mathscr{A} \lambda_{m}$ [15]. The expected number of small cells from all tiers crossed by $\mathcal{U}_{0}$ is obtained as follows.

Proposition 1 The expected number of small cells crossed by $\mathcal{U}_{0}$ can be expressed as:

$$
\mathbb{E}\left[N_{0}\right]=\sum_{m=2}^{K} \lambda_{m} \mathscr{A}_{m}
$$

where $\mathscr{A}_{m}=\frac{r_{m}}{\sqrt{\lambda_{w}}}$ is the area surrounding $\mathcal{P}_{0}$ and any small cell of the $m$ th tier is crossed by $\mathcal{U}_{0}$ if its SCBS is located in this area.

Proof: As explained earlier, the $j$ th small cell is crossed by $\mathcal{U}_{0}$ if it is located at distance less than its radius from $\mathcal{P}_{0}$. In other words, the small cell of the $m$ th tier is crossed by $\mathcal{U}_{0}$ if the BS of this small cell is located in the area $\mathcal{A}_{m}$, where $\mathcal{A}_{m}$ is the area surrounding $\mathcal{P}_{0}$. Since SCBSs are distributed as PPP, the number of SCBSs from the $m$ th tier in $\mathscr{A}_{m}$ has a Poisson distribution with mean $\lambda_{m} \mathscr{A}_{m}$ [15]. When $r_{m}=$ $r_{j, m} \forall j, \mathscr{A}_{m}=2 r_{m} \mathbb{E}\left[\left\|W_{1}-W_{0}\right\|\right]=\frac{r_{m}}{\sqrt{\lambda_{w}}}$. The result in Eq. (8) is reached.

$\mathcal{U}_{0}$ spends the pause time $S$ associated either to one of the small cells or to the first tier. This depends on the location of each waypoint. $\mathcal{U}_{0}$ spends the pause time in the coverage of small cells if the location of the destination waypoint is located in one of the small cell's coverage. Finding out where $\mathcal{U}_{0}$ spends the pause time helps to obtain the total time that $\mathcal{U}_{0}$ spends in the small cells' coverage which is also known as the potential offloading opportunity to the small cell [7]. The expectation of the pause time spent in the small cells' coverage is obtained as follows.
Lemma 2 The expected value of the time that $\mathcal{U}_{0}$ resides in any small cell during the pause time is obtained as:

$$
\mathbb{E}\left[S_{\bar{K}}\right]=\frac{\mathcal{A}_{\bar{K}}\left(s_{\max }+s_{\min }\right)}{2}
$$

Proof: $\mathcal{U}_{0}$ spends the pause time associated to one of the small cells, if any small cell is located at a distance equal to or less than the small cell's radius from the destination point $W_{1}$ :

$$
S_{\bar{K}}=S \mathbf{1}\left(\left\|W_{1}-x_{m, j}\right\| \leq r_{m, j}\right)
$$

where $x_{m, j}$ is the location of the $j$ th SCBS of the $m$ th tier. Without loss of generality, we assume that $W_{1}$ is located at the origin. The probability that this point is located in the small cells coverage is $\mathcal{A}_{\bar{K}}$ as shown in Lemma 1, then the expected pause time that $\mathcal{U}_{0}$ spends in the small cells' coverage can be expressed as:

$$
\mathbb{E}\left[S_{\bar{K}}\right]=S \mathcal{A}_{\bar{K}}
$$

The result in Eq. (9) is reached when $S$ is uniformly distributed on $\left[s_{\min }, s_{\max }\right]$.

Next, the distribution of the one-tier small cells around $\mathcal{P}_{0}$ is investigated in order to propose a framework for estimating the coverage of the small cells on $\mathcal{P}_{0}$.

\section{A. One-Tier Small Cell Network}

For enhancing the tractability, the total coverage of cells has been assumed to have a regular shape (e.g. circle) for estimating the handoff rate and the cell sojourn time in the cellular systems [4], [5], [11], [19]. This assumption holds in estimating the small cells coverage in the inter-frequency deployment, if the overlap coverage among the small cells are taken into consideration. Since the association between the first tier and the small cell tiers is based on MRPA, the coverage of the small cells is independent of the distances to the MCBSs. Therefore, it is assumed that the coverage of any small cell forms a circle (including some overlaps). The time that $\mathcal{U}_{0}$ stays associated to each small cell during its movement is essential in our analysis to derive different parameters such as the cell sojourn time. The time that $\mathcal{U}_{0}$ is associated to each small cell depends on the segment of $\mathcal{P}_{0}$ covered by this small cell. Therefore, the following investigates the coverage of each small on $\mathcal{P}_{0}$. Given that $\mathcal{U}_{0}$ crosses the $i$ th small cell that is located at distance $\tau_{i}$ from $\mathcal{P}_{0}$, the covered segment of $\mathcal{P}_{0}$ by the $i$ th small cell can be obtained as:

$$
C_{i}=\sqrt{4 r_{i}^{2}-4 \tau_{i}^{2}}, \quad \tau_{i} \leq r_{i}
$$

where $r_{i}$ is the radii of the $i$ th small cell. Since the locations of SCBSs and the waypoints are randomly distributed in the system, the segment of $\mathcal{P}_{0}$ covered by any small cell is a random variable depending on the small cell's radii and the distance between the corresponding SCBS and $\mathcal{P}_{0}$. The probability density function (PDF) of the segment of $\mathcal{P}_{0}$ covered by any small cell is derived in the following Lemma. Lemma 3 The PDF of the $i$ th small cell coverage on $\mathcal{P}_{0}$ can be expressed as:

$$
f_{C_{i}}(c)=\frac{c}{4 r_{i}^{2} \sqrt{1-\frac{c^{2}}{4 r_{i}^{2}}}}
$$


where $0 \leq c \leq 2 r_{i}$

Proof: Assuming that $\tau_{i}$ is uniformly distributed in $\left[0, r_{i}\right]$, the PDF of the coverage can be found by using the transforming density function as:

$$
\begin{aligned}
f_{C_{i}}(c) & =f_{\tau_{i}}\left(\tau\left(c_{i}\right)\right)\left|\frac{d \tau}{d c}\right| \\
& \stackrel{(a)}{=} \frac{1}{r_{i}} \frac{d}{d c}\left(\sqrt{r_{i}^{2}-\frac{c_{i}^{2}}{4}}\right)
\end{aligned}
$$

where $f_{\tau_{i}}(\tau)=1 / r_{i}$ is the PDF of the distance between the $i$ th SCBS and $\mathcal{P}_{0}$, (a) follows from Eq. (12). The result in Eq. (13) is reached after solving Eq. (14).

This result can be also defined as the PDF of the distance that $\mathcal{U}_{0}$ travels in the coverage of any small cell, which is derived under the assumption that no overlap occurs. However, the overlap coverage will be addressed later in Lemma 5 in order to obtain the accurate total coverage of the small cells on $\mathcal{P}_{0}$ and the cell sojourn time.

The expectation of the $i$ th small cell coverage on $\mathcal{P}_{0}$ can be obtained as:

$$
\mathbb{E}\left[C_{i}\right]=\int_{0}^{\infty} c_{i} f_{C_{i}}(c) d c=\int_{0}^{2 r_{i}} \frac{c^{2}}{4 r_{i}^{2} \sqrt{1-\frac{c^{2}}{4 r_{i}^{2}}}} d c
$$

the integral limits are from the maximum and the minimum coverage of any small cell with radius $r_{i}$ on $\mathcal{P}_{0}$ are $2 r_{i}$ and 0 , respectively. Since all the small cells are randomly distributed and have the same distribution around $\mathcal{P}_{0}$ and the locations of the SCSBs are uncorrelated, the expected value of the total small cells coverage on the path can be obtained by summing up all the small cells crossed by $\mathcal{U}_{0}$ (linearity of expectation). When $r=r_{i}, \forall i$, the summation of the small cells coverages can be expressed as:

$$
\mathbb{E}\left[C_{T}\right]=\mathscr{A}_{2} \lambda_{2} \mathbb{E}[C]
$$

where $\mathscr{A}_{2}=2 r \mathbb{E}\left[\left\|W_{1}-W_{0}\right\|\right]$. The result in Eq. (16) includes some overlap coverages on $\mathcal{P}_{0}$. The overlaps can be ignored when the density of small cells is very low. However, it is anticipated that the small cell density in the future cellular networks is very high and the overlap coverage needs to be taken into consideration. Some overlaps will occur on $\mathcal{P}_{0}$ and the number of these overlaps depends on various parameters such as the density of small cells and the coverage of each small cell. Finding the overlap coverage will not only help to estimate the cell sojourn time and the handoff rate precisely, but also help to estimate the energy consumption needed for the small cell discovery in the HetNets [8]. Before investigating the overlap coverage on $\mathcal{P}_{0}$, we make an assumption based on the following definition.

Definition 1 When $\mathbb{R}^{\delta}$ is a $\delta$-dimensional Euclidean space, a uniform PPP on $\mathbb{R}^{\delta} \times[0, \eta]$ of intensity $\lambda$ can be interpreted as a MPP on $\mathbb{R}^{\delta}$ with marks from $[0, \eta]$ and intensity $\eta \lambda[20]$.

Assumption 1: Without loss of generality, if $r_{j}=r, \forall j$ and the point $W_{0}$ is at the origin, the SCBSs at distance of $r$ or less from the line that starts from the origin and passes through $W_{1}$, can be interpreted as a MPP on $\mathbb{R}^{+} \times[0, r]$, $\bar{\Phi}=\left\{\left(\bar{y}_{i}, \tau_{i}\right)\right\}$ of intensity $\bar{\lambda}$, where $\bar{y}_{i}$ is the path point that represents the nearest point on the line to the SCBS of the $i$ th crossed small cell as shown in Fig. 2. The path points are assumed to be distributed on the line as PPP. The accuracy of this assumption is validated through simulations in Fig. 3. $\tau_{i}$ represents the vertical distance from the location of the $i$ th SCBS to the path point $\bar{y}_{i}$. Since the locations of the SCBSs are uniformly distributed and can be at any distance from $\mathcal{P}_{0}$, it is also assumed that $\tau$ is uniformly distributed in the range $[0, r]$. Since the small cells crossed by $\mathcal{U}_{0}$ is mapped to MPP, the density of the new process needs to be investigated as it directly affects different parameters in our analysis regarding the overlaps among the small cells, the handoff rate and the cell sojourn time. Therefore, the following Lemma addresses the density of the new MPP as:

Lemma 4 The density of the MPP on the straight line from $W_{0}$ and passing through $W_{1}$ can be expressed as:

$$
\bar{\lambda}=2 r \lambda
$$

Proof: It is assumed that the expected number of SCBSs in the area $2 r L$ is $\mathscr{N}$. According to Assumption 1, the number of the path points $\overline{\mathscr{N}}=\mathscr{N}$ :

$$
\begin{aligned}
& \overline{\mathscr{N}}=\mathscr{N} \\
& \bar{\lambda} L=2 r L \lambda
\end{aligned}
$$

The result in Eq. (17) is reached.

The path points are set in order according to the distance from $W_{0}$ as $\left(\bar{y}_{1}, \bar{y}_{2}, \cdots, \bar{y}_{i}, \cdots\right)$. The path point inter-distance (e.g. the first path point inter-distance represents the distance between the points $\bar{y}_{1}$ and $\bar{y}_{2}$ ) has an exponential distribution with $\bar{\lambda}$ :

$$
\mathbb{P}\left(\left\|\bar{y}_{i+1}-\bar{y}_{i}\right\| \leq \mathrm{d}\right)=1-\exp (-\bar{\lambda} \mathrm{d}) \quad \mathrm{d}>0
$$

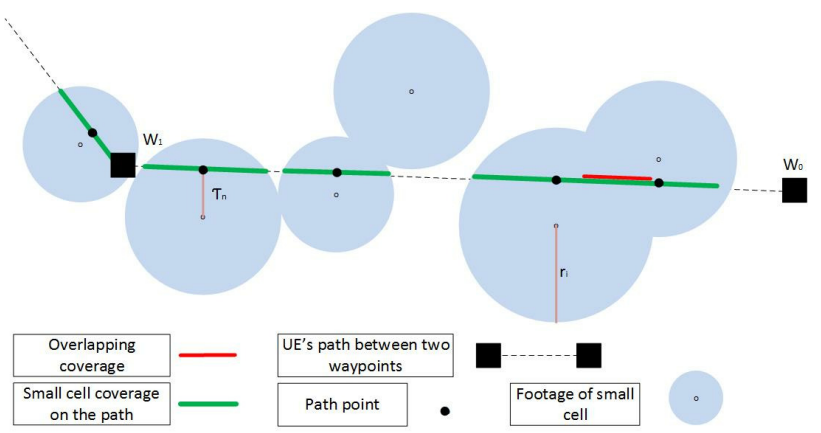

Fig. 2: Coverage of small cells from different tiers on $\mathcal{P}_{0}$.

Proposition 2 The expected value of the distance from $W_{0}$ to $\bar{y}_{i}$ can be expressed as:

$$
\mathbb{E}\left(\left\|\bar{y}_{i}-W_{0}\right\|\right)=\frac{i}{\bar{\lambda}}
$$

Proof: Since the path points $\bar{y}$ follow PPP on $\mathbb{R}^{+}$, the distance $\left\|\bar{y}_{i}-W_{0}\right\|$ for $i>0$ has an Erlang or Gamma distribution with $i$ and $\bar{\lambda}$. The PDF of $\left\|\bar{y}_{i}-W_{0}\right\|$ is expressed as:

$$
f_{\left(\left\|\bar{y}_{i}-W_{0}\right\|\right)}(\mathrm{d})= \begin{cases}\frac{\bar{\lambda}^{i}}{\Gamma(i)} \mathrm{d}^{i-1} e^{-\bar{\lambda} \mathrm{d}}, & \mathrm{d}>0 \\ 0, & \text { otherwise }\end{cases}
$$




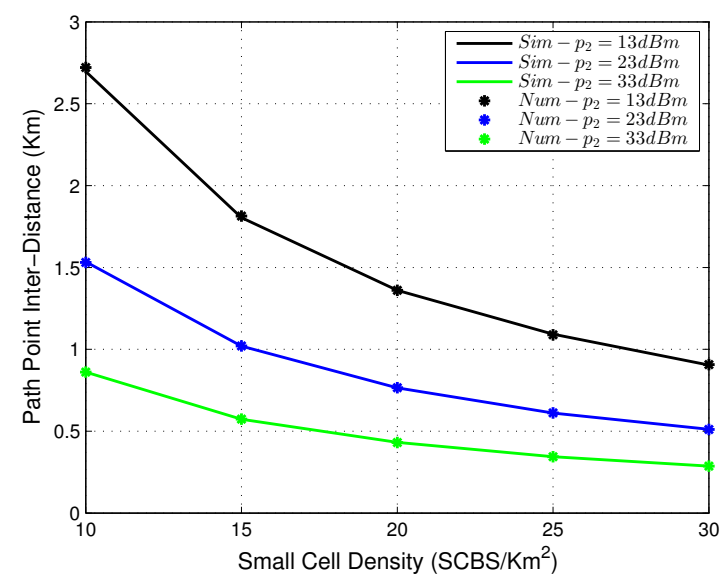

Fig. 3: Assumption 1 is validated in single-tier small cells.

where $\Gamma($.$) represents the gamma function. The expectation of$ $\left\|\bar{y}_{i}-W_{0}\right\|$ is obtained as:

$$
\mathbb{E}\left[\left\|\bar{y}_{i}-W_{0}\right\|\right]=\int_{0}^{\infty} \mathrm{d} f_{\left(\left\|\bar{y}_{i}-W_{0}\right\|\right)}(\mathrm{d}) d \mathrm{~d}=\frac{\bar{\lambda}^{i} \Gamma(i+1)}{\Gamma(i) \bar{\lambda}^{i+1}}
$$

where $\frac{\Gamma(i+1)}{\Gamma(i)}=i$ and the result in Eq. (20) is reached.

Lemma 5 The expected value of one overlap coverage on $\mathcal{P}_{0}$ can be obtained as:

$$
\mathbb{E}\left[\mathfrak{C}_{i}\right]=\frac{\mathbb{E}\left[\bar{C}_{i}\right]}{2}
$$

where $\bar{C}_{i}=\frac{C_{i}}{2}+\frac{C_{i+1}}{2}$ represents the maximum distance between the $i$ th path point and the $(i+1)$ th path point for the $i$ th overlap to take place and, $\frac{C_{i}}{2}$ and $\frac{C_{i+1}}{2}$ are the half of the total coverage of the $i$ th and the $(i+1)$ th small cells on $\mathcal{P}_{0}$ as shown in Fig. 4.

Proof: See Appendix A.

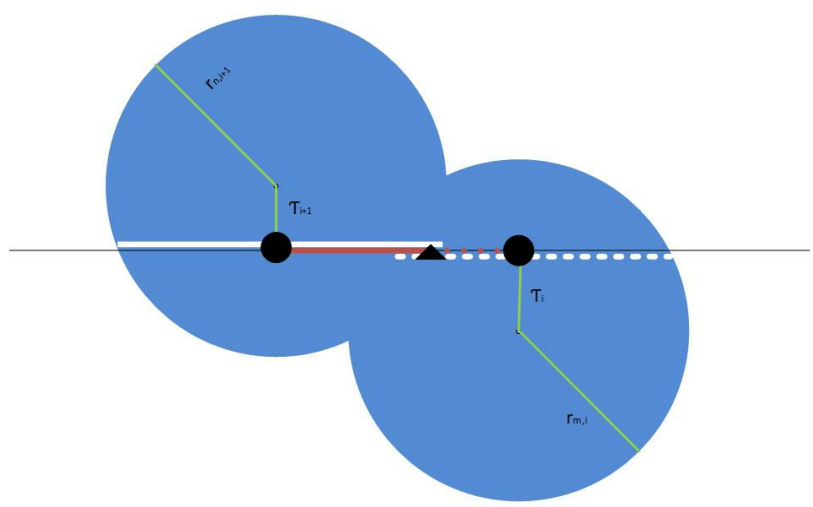

Fig. 4: The overlap coverage between two small cells. The white dashed and solid lines represent the footage coverage of $i$ th and $(i+1)$ th small cells on UE's path $\left(C_{m, i}, C_{n, i+1}\right)$, the red dashed and solid lines represent the one side actual coverage of $i$ th and $(i+1)$ th small cells on UE's path $\left(\Psi_{m, i}, \Psi_{n, i+1}\right)$, the black circle represents the path point and black triangle represents the point where the received signals from $i$ th and $(i+1)$ th small cells are equal $(O)$.

After finding the expected value of any overlap coverage on $\mathcal{P}_{0}$ in Lemma 5 , the expected number of overlaps taking place on $\mathcal{P}_{0}$ is obtained as follows.
Lemma 6 The expected number of overlaps on $\mathcal{P}_{0}$ can be expressed as:

$$
\mathbb{E}\left[N_{O L}\right]=\mathbb{E}\left[N_{O L, \max }\right]\left(1-e^{-\bar{\lambda} \int_{0}^{4 r} \bar{c} f_{\bar{C}_{i}}(\bar{c})}\right)
$$

where $\mathbb{E}\left[N_{O L, \text { max }}\right]=\mathcal{A}_{1}\left(\frac{\bar{\lambda}}{2 \sqrt{\lambda_{w}}}-1\right)+\frac{\mathcal{A}_{\bar{K}}}{2}\left(\frac{\bar{\lambda}}{\sqrt{\lambda_{w}}}-1\right)$ is the expectation of the maximum overlaps can occur.

Proof: See Appendix B.

The expectation of the actual small cells coverage on $\mathcal{P}_{0}$ can be obtained as:

$$
\mathbb{E}\left[\mathcal{C}_{S C}\right]=\mathbb{E}\left[C_{T}\right]-\mathbb{E}\left[C_{O L}\right]
$$

where $\mathbb{E}\left[C_{O L}\right]=\mathbb{E}\left[N_{O L}\right] \mathbb{E}\left[\mathfrak{C}_{i}\right]$ is the expectation of the overlap coverage on $\mathcal{P}_{0}$.

\section{B. Multi-Tier Small Cell Network}

In this subsection, we expand the one-tier small cell framework proposed in the previous subsection to a multi-tier small cell framework. Now we need to consider different densities and small cell footages. The analysis in this subsection will be based on the following definition.

Definition 2 Superposition of two independent PPPs is a PPP with intensity of the sum of both densities [15].

From Definition 2, the small cells from different tiers in the system form one PPP $\Phi_{\bar{K}}$ with density of $\lambda_{\bar{K}}=\sum_{m=2}^{K} \lambda_{m}$. Therefore, the small cells crossed by $\mathcal{U}_{0}$ from different tiers can also be interpreted as one MPP on $\mathbb{R}^{+} \times\left[0, r_{m}\right], \bar{\Phi}_{\bar{K}}=$ $\left\{\left(\bar{z}_{i}, \tau_{i}\right)\right\}$ of intensity $\bar{\lambda}_{\bar{K}}$ where $r_{m}$ takes a value in the range $\left[r_{2}, r_{3}, \cdots, r_{m}, \cdots, r_{K}\right]$. The density of path points on the straight line from the origin can be found similar to Lemma 4 as:

$$
\begin{aligned}
\bar{\lambda}_{\bar{K}} & =\sum_{m=2}^{K} \bar{\lambda}_{m} \\
& =\sum_{m=2}^{K} 2 r_{m} \lambda_{m}
\end{aligned}
$$

The path points on $\mathcal{P}_{0}$ are set in order $\left(\bar{z}_{1}, \bar{z}_{2}, \cdots, \bar{z}_{i}, \cdots\right)$. The path points inter-distance has an exponential distribution with density $\bar{\lambda}_{\bar{K}}$ :

$$
\mathbb{P}\left(\left\|\bar{z}_{i}-\bar{z}_{i+1}\right\| \leq d\right)=1-\exp \left(-\bar{\lambda}_{\bar{K}} d\right) \quad d>0
$$

Given a small cell of the $m$ th tier overlaps with another small cell on $\mathcal{P}_{0}$, the expected value of the overlap coverage can be obtained similar to Lemma 5 as follows.

Lemma 7 The expected value of one overlap occurring between the $i$ th small cell of the $m$ th tier and the $(i+1)$ th small cell of the $n$th tier, can be found as:

$$
\mathbb{E}\left[\mathfrak{C}_{m n}\right]=\frac{\sum_{n=2}^{K} \bar{\lambda}_{n} \mathbb{E}\left[\bar{C}_{m n}\right]}{2 \bar{\lambda}_{\bar{K}}}
$$

where $\bar{C}_{m n}=\frac{C_{m, i}}{2}+\frac{C_{n, i+1}}{2}$ is the maximum distance between $\bar{z}_{i}$ and $\bar{z}_{i+1}$ for an overlap to occur between the $i$ th small cell of the $m$ th tier and the $(i+1)$ th small cell of the $n$th tier, $\frac{C_{m, i}}{2}\left(\frac{C_{n, i+1}}{2}\right)$ is the half coverage of the $i$ th $((i+1)$ th $)$ small 
cell of the $m$ th ( $n$ th) tier on $\mathcal{P}_{0}$, and $\frac{\bar{\lambda}_{n}}{\bar{\lambda}_{\bar{K}}}$ is the probability of the $(i+1)$ th small cell being from the $n$th tier.

Proof: See Appendix C.

In the multi-tier small cell network, there are different small cells with different footages and different densities crossed by $\mathcal{U}_{0}$ during its movement. Since the path points follow a PPP on $\mathcal{P}_{0}$, the probability of a given small cell of the $m$ th tier overlapping with another small cell on $\mathcal{P}_{0}$ can also be found from the null probability and similar to Lemma 6 as:

$$
\begin{aligned}
\mathbb{P}_{O L, m n} & =1-\prod_{n=2}^{K} \mathbb{P}\left[\left\|\bar{z}_{n, i+1}-\bar{z}_{m, i}\right\|>\mathbb{E}\left[\bar{C}_{m n}\right]\right] \\
& \left.=1-\exp \left(-\sum_{n=2}^{K} \bar{\lambda}_{n} \int_{0}^{2 r_{m}+2 r_{n}} \bar{c} f_{\bar{C}_{m n}}(\bar{c})\right]\right)
\end{aligned}
$$

where $\exp \left(-\sum_{n=2}^{K} \bar{\lambda}_{n} \int_{0}^{2 r_{m}+2 r_{n}} \bar{c} f_{\bar{C}_{m n}}(\bar{c})\right)$ represents the probability of that a small cell from the $m$ th tier does not overlap with another small cell on $\mathcal{P}_{0}$. The expected number of overlaps can be expressed as:

$$
\mathbb{E}\left[N_{O L}\right]=\mathbb{E}\left[N_{O L, \max }\right] \frac{\sum_{m=2}^{K} \bar{\lambda}_{m} \mathbb{P}_{O L, m n}}{\bar{\lambda}_{\bar{K}}}
$$

where $\frac{\bar{\lambda}_{m}}{\bar{\lambda}_{\bar{K}}}$ is the probability of the $i$ th small cell being from the $m$ th tier on the path. The expectation of the actual time that $\mathcal{U}_{0}$ spends in the small cells' coverage are obtained as:

$$
\mathbb{E}\left[\mathcal{T}_{S C}\right]=\frac{\mathbb{E}\left[C_{T}\right]-\mathbb{E}\left[C_{O L}\right]}{v}+\mathbb{E}\left[S_{\bar{K}}\right]
$$

where $\mathbb{E}\left[C_{T}\right]=\sum_{m=2}^{K} \frac{\bar{\lambda}_{m} \mathbb{E}\left[C_{m}\right]}{2 \sqrt{\lambda_{w}}}, \mathbb{E}\left[C_{O L}\right]=$ $\sum_{m=2}^{K} \mathbb{E}\left[N_{O L}\right] \mathbb{E}\left[\mathfrak{C}_{m n}\right]$ and $\mathbb{E}\left[S_{\bar{K}}\right]$ is obtained in Lemma 2 . The result in Eq. (31) shows that the total coverage does not only depend on the second tier cell association and the total transition time as it was assumed in [7], but also depends on other system parameters such as transmit power and the density of each tier as well as the probability of an overlap occurring on $\mathcal{P}_{0}$.

\section{SoJourn Time}

The cell sojourn time is defined as the expected time that $\mathcal{U}_{0}$ stays in a cell coverage of interest and it directly affects the efficiency of system resources utilization. Since all transition lengths are i.i.d, the expected sojourn time will be derived during one transition time (e.g. $\left.\mathcal{P}_{0}\right)$.

\section{A. Small Cell Sojourn Time}

The small cells crossed by $\mathcal{U}_{0}$ have different coverages on $\mathcal{P}_{0}$ as they have different transmit powers and they are located at different distances from $\mathcal{P}_{0}$. Since the cell association among the small cells is based on the maximum received power, the overlap coverages will be served by different small cells depending on the transmit powers and the locations of the SCBSs around the path. The expectation of the $i$ th small cell's footage from the $m$ th tier, served by the $(i+1)$ th small cell from the $n$th tier due to overlapping is obtained as follows.

Lemma 8 Given that an overlap occurs on the path between the $i$ th small cell of the $m$ th tier and the $(i+1)$ th small cell of the $n$th tier, the expected value of the $i$ th small cell's footage served by the $(i+1)$ th small cell due to overlapping is expressed as:

$$
\mathbb{E}\left[\chi_{n \mapsto m}\right]=\frac{\mathbb{E}\left[\mathfrak{C}_{m n}\right]\left(\frac{p_{m}}{p_{n}}\right)^{\frac{-1}{\alpha_{m}}}}{1+\left(\frac{p_{m}}{p_{n}}\right)^{\frac{-1}{\alpha_{m}}}}
$$

Proof: See Appendix D.

The expectation of the small cell sojourn time when the pause time is zero can be expressed in the next Theorem.

Theorem 1 When $\lambda_{w}$ is small, $V=v$ and $S=0$, the average small cell sojourn time during one movement can be expressed as:

$$
\begin{aligned}
\mathbb{E}\left[S \mathcal{T}_{S C S}^{0}\right]=\sum_{m=1}^{\bar{K}} \frac{\bar{\lambda}_{m}}{\bar{v} \lambda_{\bar{K}}}\left(\mathbb{E}\left[C_{m}\right]\right. & -\sum_{n=1}^{\bar{K}} \frac{\mathbb{P}_{O L, m n} \bar{\lambda}_{n}}{\bar{\lambda}_{\bar{K}}} \mathbb{E}\left[\chi_{n \mapsto m}\right] \\
& \left.-\sum_{q=1}^{\bar{K}} \frac{\mathbb{P}_{O L, m q} \bar{\lambda}_{q}}{\bar{\lambda}_{\bar{K}}} \mathbb{E}\left[\chi_{q \mapsto m}\right]\right)
\end{aligned}
$$

where $\mathbb{P}_{O L, m n}\left(\mathbb{P}_{O L, m q}\right)$ is the probability that the reference small cell from the $m$ th tier overlaps with the $(i+1)$ th $((i-$ 1 )th) small cell from the $n$th $\left(q\right.$ th) tier on $\mathcal{P}_{0}$ and is obtained in Eq. (29).

Proof: See Appendix E.

Before deriving the small cell sojourn time in a multi-tier small cell network when $S \neq 0$, we give the probability of $\mathcal{U}_{0}$ spending the pause time in the coverage of the reference small cell of the $m$ th tier as follows.

Lemma 9 The probability that $\mathcal{U}_{0}$ spends the pause time in the reference small cell of the $m$ th tier is obtained as:

$$
\mathbb{P}_{\mathcal{S} \mathcal{T}, m}=\frac{2 \pi\left(\frac{\rho_{\text {min }}}{\mathscr{L}_{2} p_{m}}\right)^{-\frac{2}{\alpha_{m}}}}{\mathscr{A}_{m}\left(\sum_{n=2}^{K}\left(\frac{p_{n}}{p_{m}}\right)^{\frac{2}{\alpha_{n}}}+1\right)}
$$

Proof: See Appendix F.

The expectation of the cell sojourn time that $\mathcal{U}_{0}$ spends in any small cell during one movement can be obtained as follows.

Theorem 2 When $\lambda_{w}$ is small, $V=v$ and $S \neq 0$, the average small cell sojourn time during one movement can be expressed as:

$$
\mathbb{E}\left[\mathcal{S} \mathcal{T}_{S C S}^{s}\right]=\sum_{m=1}^{\bar{K}} \frac{\bar{\lambda}_{m}}{\bar{\lambda}_{\bar{K}}}\left(\mathcal{A}_{\bar{K}} \mathbb{E}\left[\mathcal{S} \mathcal{T}_{m}^{s}\right]+\mathcal{A}_{1} \mathbb{E}\left[\mathcal{S} \mathcal{T}_{m}^{0}\right]\right)
$$

where $\mathbb{E}\left[S \mathcal{T}_{m}^{s}\right]=\mathbb{P}_{\mathcal{S} \mathcal{T}, m}\left(\mathbb{E}[S]+\mathbb{E}\left[\mathcal{S} \mathcal{T}_{m}^{0}\right]\right)+(1-$ $\left.\mathbb{P}_{\mathcal{S T}, m}\right) \mathbb{E}\left[\mathcal{S T}_{m}^{0}\right]$ is the expectation of the $m$ th cell sojourn time when $S \neq 0$ and $\mathbb{E}\left[S \mathcal{S T}_{m}^{0}\right]$ is obtained in Eq. (E5).

Proof: When $W_{1}$ is located in the small cells coverage with a probability of $\mathcal{A}_{\bar{K}}, \mathcal{U}_{0}$ spends the pause time in the small cell coverage of the $m$ th tier with a probability of $\mathbb{P}_{\mathcal{S}} \mathcal{T}_{m}$.

The above results are more suitable for small values of $\lambda_{w}$ as $\mathcal{U}_{0}$ is expected to cross a number of small cells during one movement. The larger values of $\lambda_{w}$ are suitable for users walking [11]. The small cell sojourn time when the actual 
coverage of each small cell is assumed to have a hexagonal shape (this assumption does not affect the accuracy of the analysis [10]) can be obtained as:

$$
\mathbb{E}\left[S \mathcal{T}_{S C L}\right]=\mathbb{E}\left[\mathcal{T}_{T}\right] \int_{0}^{2 \pi} \int_{0}^{Q} \tilde{f}(\ell, \theta) d \ell d \theta
$$

where $\mathbb{E}\left[\mathcal{T}_{T}\right]=\mathbb{E}\left[T_{0}\right]+\mathbb{E}[S], Q=\sum_{m=1}^{\bar{K}} \frac{\bar{\lambda}_{m}}{\bar{\lambda}_{\bar{K}}}\left(r_{m}-\right.$ $\left.\sum_{n=1}^{\bar{K}} \frac{\mathbb{P}_{O L, m n} \lambda_{n}}{\lambda_{\bar{K}}} \mathbb{E}\left[\chi_{n \mapsto m}\right]\right)$ is found similar to Lemma 8 and $\tilde{f}(\ell, \theta)$ is the UE distribution between $W_{0}$ and $W_{1}$ for the incremental space $d A(\ell, \theta)$ around the point $(\ell, \theta)$ [11], [21].

\section{B. Macro Cell Sojourn Time}

Deploying dense small cells in the network will not only affect the small cell sojourn time but also affect the macro cell sojourn time. Since the macro cells form VTCs, the expectation of the macro cell sojourn time are obtained as:

$$
\begin{array}{r}
\mathbb{E}\left[\mathcal{S} \mathcal{T}_{M C}\right]=\frac{\mathbb{E}\left[\mathcal{T}_{T}\right]}{\bar{\lambda}_{\bar{K}}+\lambda_{M C B}}\left(\lambda_{M C B} \int_{0}^{2 \pi} \int_{0}^{R_{M C}} f(\ell, \theta) d \ell d \theta\right. \\
\left.+\bar{\lambda}_{\bar{K}} \int_{0}^{2 \pi} \int_{0}^{\mathcal{D}_{S C}} f(\ell, \theta) d \ell d \theta\right)
\end{array}
$$

where $R_{M C}$ is the average macro cell radius, $\lambda_{M C B}=$ $\frac{2}{\sqrt{\pi} R_{M C}}$ is the length intensity of the macro-macro boundaries [13], $f(\ell, \theta)$ is the spatial UE distribution when $S=0$ [11], $\frac{\lambda_{M C B}}{\bar{\lambda}_{\bar{K}}+\lambda_{M C B}}\left(\frac{\lambda_{\bar{K}}}{\bar{\lambda}_{\bar{K}}+\lambda_{M C B}}\right)$ represents the probability that $\mathcal{U}_{0}$ reaches the macro-macro (macro-small) boundaries before the macro-small (macro-macro) boundaries, and $\mathcal{D}_{S C}$ is the expectation of the distance to the nearest small cell coverage on the path and obtained as:

$$
\begin{aligned}
\mathcal{D}_{S C} & =\int_{0}^{\infty} l \frac{d}{d l}\left(1-\exp \left(-\bar{\lambda}_{\bar{K}}\left(l-\sum_{m=2}^{K} \frac{\bar{\lambda}_{m} \mathbb{E}\left[C_{m}\right]}{2 \bar{\lambda}_{\bar{K}}}\right)\right) d l\right. \\
& =\int_{0}^{\infty} l \bar{\lambda}_{\bar{K}} \exp \left(-\bar{\lambda}_{\bar{K}} l+\sum_{m=2}^{K} \frac{\bar{\lambda}_{m} \mathbb{E}\left[C_{m}\right]}{2}\right) d l \\
& =\frac{\exp \left(\sum_{m=2}^{K} \frac{\bar{\lambda}_{m} \mathbb{E}\left[C_{m}\right]}{2}\right)}{\bar{\lambda}_{\bar{K}}}
\end{aligned}
$$

where $\left(1-\exp \left(-\bar{\lambda}_{\bar{K}}\left(l-\sum_{m=2}^{K} \frac{\bar{\lambda}_{m} \mathbb{E}\left[C_{m}\right]}{2 \bar{\lambda}_{\bar{K}}}\right)\right)\right.$ is the probability of that the first path point of the small cell with an expected coverage of $\sum_{m=2}^{K} \frac{\bar{\lambda}_{m} \mathbb{E}\left[C_{m}\right]}{\bar{\lambda}_{\bar{K}}}$ is at distance greater than $l$.

\section{HANDOFF RATE}

The handoff rate is defined as the expected number of handoffs taking place per unit time. It is considered as one of the important parameters in the cellular systems as it affects the amount of signalling. Increasing the number of UEs and the number of small cells in the system will affect the amount of signalling significantly and also affect the QoS. It is also anticipated that the first tier will have to deal with a big number of UEs, therefore, an accurate framework will help in network dimensioning and also to estimate the required resources at the first tier. We define the IRH rate and the IAH rate to reflect the required resources at the first tier. The IRH is defined as the number of handoffs taking place between two cells of different frequencies (e.g. small cell to macro cell and/or macro cell to small cell). The IAH is defined as the number of handoffs taking place between two cells of the same frequency (e.g. macro cell to macro cell or/and small cell to small cell). In this paper, we consider the IAH rate among the small cells since the handoff rate in the first tier was already studied in [11]. The expected handoff rate in a multi-tier HetNet is defined as the expected number of handoffs during one movement.

$$
\mathbb{E}\left[\mathcal{H}_{T}\right]=\mathbb{E}\left[\mathcal{H}_{I R}\right]+\mathbb{E}\left[\mathcal{H}_{I A}\right]=\frac{\mathbb{E}\left[N_{H F}\right]}{\mathbb{E}\left[T_{0}\right]}
$$

where $N_{H F}$ represents the number of handoffs that $\mathcal{U}_{0}$ experiences during its movement along $\mathcal{P}_{0}$, and $\mathcal{H}_{I R}$ and $\mathcal{H}_{I A}$ represent the IRH rate and the IAH rate respectively. $\mathcal{U}_{0}$ can experience up to $2 N_{0}$ handoffs during its movement (maximum of $2 N_{0}$ handoffs take place when there is no overlap on $\mathcal{P}_{0}$ ). The expectations of both IRH rate and IAH rate are obtained as follows.

Theorem 3 The expected IRH rate can be expressed as:

$$
\mathbb{E}\left[\mathcal{H}_{I R}\right]=\frac{2}{\mathbb{E}\left[T_{0}\right]}\left(\mathbb{E}\left[N_{0}\right]-\mathbb{E}\left[N_{O L}\right]\right)
$$

where $\mathbb{E}\left[N_{O L}\right]$ is obtained in Eq. (30).

Proof: The IAH rate is defined as the number of handoffs between cells operating on the same frequency during one movement divided by the total time of movement. Since the IAH takes place on the high frequency $F_{2}$ when $\mathcal{U}_{0}$ moves between small cells overlapped on $\mathcal{P}_{0}$. Therefore the number of IAHs can also be interpreted as the total number of small cells overlapping on $\mathcal{P}_{0}$. The maximum number of handoffs that $\mathcal{U}_{0}$ can experience on $\mathcal{P}_{0}$, is $2 N_{0}$. Since some of the handoffs will be between two small cells due to overlapping, the total number of handoffs can be expressed as $2 N_{0}-N_{O L}$. Therefore the expectation of the total handoff rate becomes:

$$
\mathbb{E}\left[\mathcal{H}_{T}\right]=\frac{2 \mathbb{E}\left[N_{0}\right]-\mathbb{E}\left[N_{O L}\right]}{\mathbb{E}\left[T_{0}\right]}
$$

The desired result in Eq. (40) is reached after substituting Eq. (41) in Eq. (39).

\section{NUMERICAL RESULTS}

In this section, simulation and numerical results are presented to validate the analysis and to show the impact of different parameters such as the waypoint density $\lambda_{w}$, the transmit power of small cells, the number of small cell tiers and the density of the small cells in the system on the handoff rate and the cell sojourn time. Some figures in this section include two scenarios. The first scenario (A) represents the analysis in this paper which considers the overlaps among the small cells. The second scenario (B) represents the analysis when the overlaps are ignored. The different system parameters can be found in Table I, unless given otherwise.

Fig. 6 and Fig. 5 show the accuracy of the analysis in this paper. The expectation of the path points inter-distance is shown when a different number of small cell tiers and different values of the small cell density are considered in the system. 
TABLE I: System Parameters

\begin{tabular}{l|c|l} 
Parameter & Symbol & Value \\
\hline \hline Minimum received power & $\rho_{\min }$ & $-90 \mathrm{dBm}$ \\
Average macro cell radius & $R_{M C}$ & $1 \mathrm{Km}$ \\
Path-loss exponent & $\alpha_{k}$ & 4 \\
Number of small cell tiers & $\bar{K}$ & 1 (default), 2 and 3 \\
Small cell transmit power & $p_{m}$ & 30,33 and $36 \mathrm{dBm}$ \\
Waypoint density & $\lambda_{w}$ & 0.01 waypoint $/ \mathrm{Km}^{2}$ \\
UE speed & $\mathrm{V}$ & $5 \mathrm{Km} / \mathrm{h}$ \\
Low frequency & $f_{1}$ & $2 \mathrm{Ghz}$ \\
High frequency & $f_{2}$ & $10 \mathrm{Ghz}$ \\
Pause time & $S$ & 0
\end{tabular}

Fig. 5 shows that the assumption of the path points forming a PPP with density of $\sum_{m=1}^{\bar{K}} 2 r_{m} \lambda_{m}$ is very accurate. It is also shown that the path points inter-distance decreases when the density of small cells increases for a different number of tiers. This is because the distances between the small cells in the system are minimized and $\mathcal{U}_{0}$ crosses more small cells during its movement.

Fig. 6 shows the total coverage of the small cells on $\mathcal{P}_{0}$

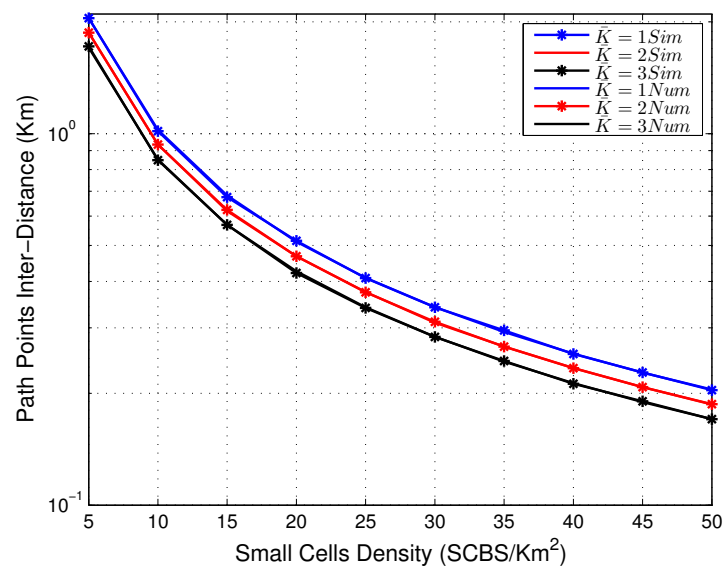

Fig. 5: The path points inter-distance.

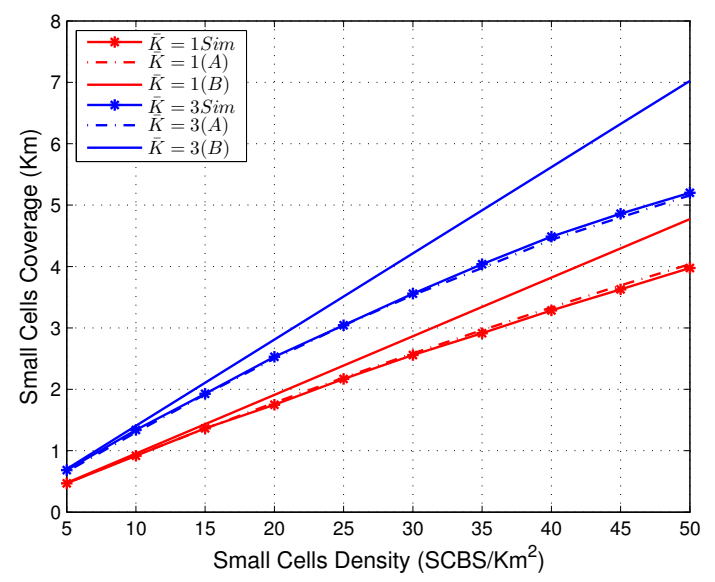

Fig. 6: The small cells coverage.

in both scenarios (A) and (B) for a different number of tiers and different values of the small cell density. The coverage of the small cells on $\mathcal{P}_{0}$ increases when the density of the small cells increases in both scenarios. However, the coverage of the small cells in (B) is doubled when the density of small cells is doubled, while the pace of increment slows down in (A) when the density increases due to overlapping. As a result, the gap between the result in (A) and the result in (B) increases when the density of the small cells increases for a different number of tiers. On the other hand, it is shown that the gap between (A) and (B) is greater when $K=4$ for the same small cell density, as the small cells with larger coverage are considered when $K=4$. It is more likely to have small cells overlapped when the radius of these cells increases. Fig. 6 also shows that the overlap coverage can be neglected when the density of small cells is very low, however, the overlap coverage increases in the dense networks and ignoring the overlap coverage will affect the accuracy significantly.

This figure can also provide insightful information regarding the trend of the coverage in HetNets, and how the density of cells and number of small cell tiers affect the coverage. It is shown that the coverage of small cells crossed by UEs (or the time that the UEs spend in the small cells' coverage) will not be doubled when the density of small cells is doubled in dense networks. This is because more overlaps are likely to take place when the density increases and/or the footprints of small cells increase. Therefore, the system designers and operators need to take this information into consideration. This figure also shows the total time that the UE spends in the small cells' coverage during its movement, which is also known as the potential offloading opportunity to the small cells [7][9]. These results can be guidelines not only for operators when considering expansion but also for estimating the total offloading opportunity to the small cells in order to choose the appropriate value of the inter-frequency scan to achieve the best system performance.

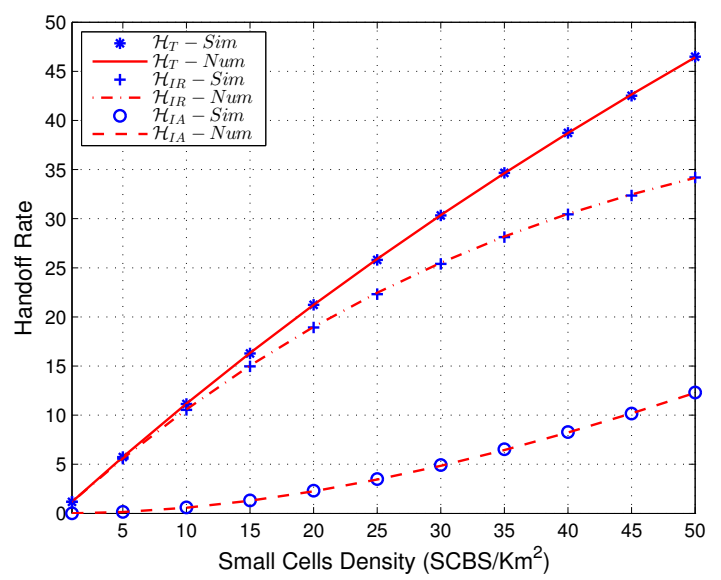

Fig. 7: IRH, IAH, and total handoff $(T H)$ rates, $\bar{K}=3$ and $\lambda_{w}=0.001$

Fig. 7 shows the total handoff rate, the IRH rate and the IAH rate for different values of the small cell density when $\bar{K}=3$. As expected, it is shown that the total handoff rate increases when the density of the small cells increases as the UEs will cross more small cells in the dense small cell network. Fig. 7 also shows the impact of the small cell density on the IAH rate and IRH rate. For low small cell density, most of the handoffs are IRHs from or to the first tier (macro cells). Although increasing the small cell density will maximize the total handoff rate, it turns some of these handoffs to IAHs due to some overlaps. Increasing the IAH rate means more handoffs and their cost (e.g. signaling) will be handled by the other tiers. 
When ignoring the overlaps on the path, all the IAHs will be counted as IRHs and the accuracy of the total handoff rate will be affected significantly in the high dense small cell network. Fig. 8 shows a comparison between the scenario when the overlaps are taken into account (A), and the scenario of ignoring the overlaps (B) on $\mathcal{P}_{0}$. The total handoff rate in (B) is always greater, as the overlaps are ignored and two IRHs are assumed to take place for each small cell (one IRH for entering the small cell and another one for leaving it). In fact, one IRH or no IRH is required when $\mathcal{U}_{0}$ moves among two or three overlapped small cells.

The number of handoffs for different values of the small cell density and different values of the mobility parameter $\lambda_{w}$ are shown in Fig. 9. It is shown that the total number of handoffs during one movement increases when the mobility parameter decreases. This is because the expected distance that $\mathcal{U}_{0}$ needs to travel from the starting point to the destination point decreases when $\lambda_{w}$ increases as shown in Eq. (6). It can also be seen from Fig. 9 that the number of IRHs for different values of $\lambda_{w}$ starts increasing dramatically with low and medium small cell density while the number of IAHs increases slightly with low and medium density of small cells. However, the number of IRHs starts dropping with high density of small cells around the density of 65 for all values of $\lambda_{w}$. It can also be seen that the higher value of $\lambda_{w}$, the more gradually the number of IRHs decreases. The number of IAHs keeps increasing until it exceeds the number of IRHs. Note that IAHs exceeds IRHs at different small cell densities when different values of $\lambda_{w}$ are considered. For instance, the number of IAHs exceeds the number of IRHs at a small cell density of 75 when $\lambda_{w}=0.01$, while the number of IAHs exceeds the number of IRHs at a small cell density of 90 when $\lambda_{w}=1$. This is because the effect of the tier association on the number of IAHs and IRHs is greater when the number of the crossed small cells is smaller (greater value of $\lambda_{w}$ ), since the number of overlaps on the path is a function of the cell association as shown in Eq. (24). Fig. 10 illustrates the

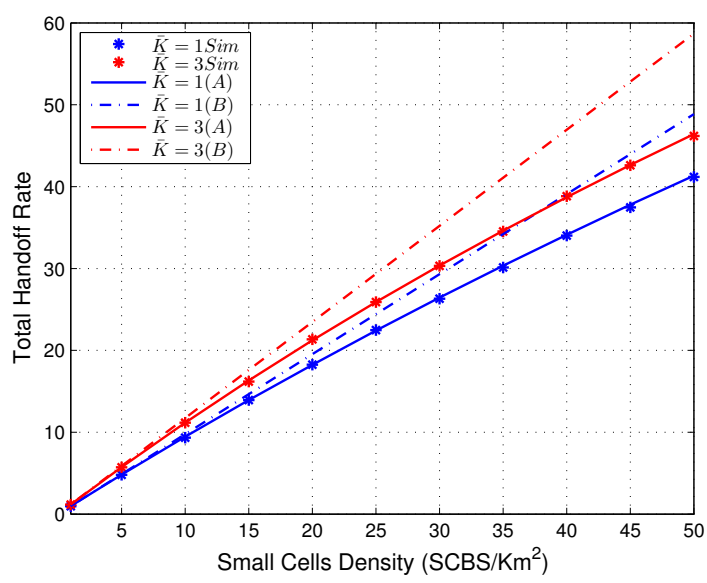

Fig. 8: Total handoff rate where $\lambda_{w}=0.001$

fraction of the handoffs that the first tier is involved, which is expressed as the ratio of the IRH rate to the total handoff rate for different transmit powers. The fraction of handoffs that the first tier needs to handle decreases when the density of small cells increases, for instance, when $p_{2}=37 \mathrm{dBm}$, less than $65 \%$ of the handoffs will be managed by the first tier when $\lambda_{2}=50$, while over $80 \%$ and $95 \%$ of the handoffs will be managed by the first tier when $\lambda_{2}=25$ and $\lambda_{2}=5$ respectively. Fig. 10 also shows that when the footages of the small cells increase, the fraction of the handoff managed by the first tier decreases. This is because more overlaps are more likely to occur when the footages of the small cells are larger for the same small cell density.

From a network perspective, the number of handoffs or

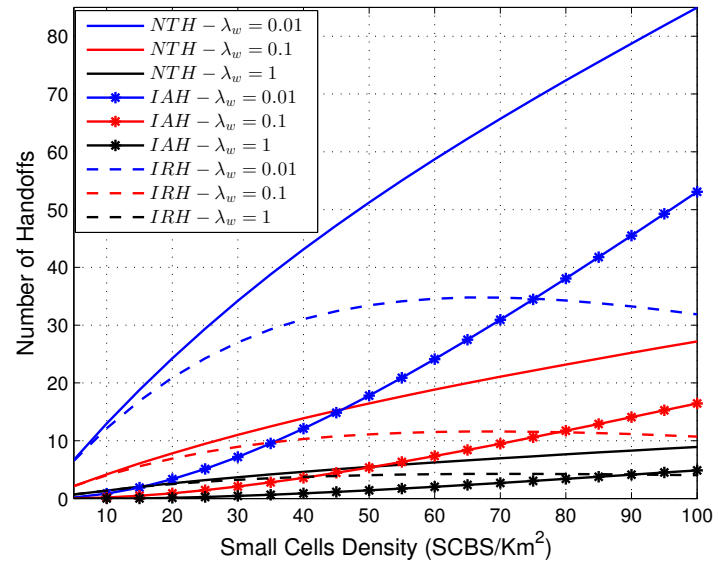

Fig. 9: Number of IAHs, IRHs and total handoffs, $\bar{K}=3$.

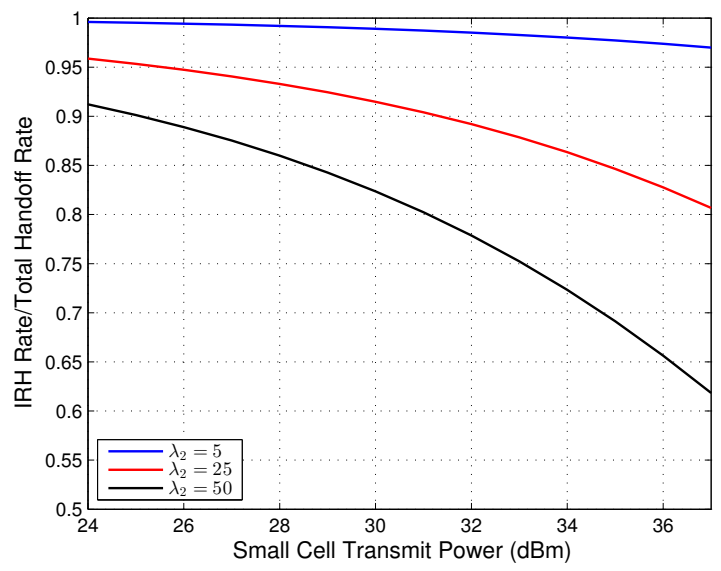

Fig. 10: The ratio of IRH rate to total handoff rate.

the signaling load required for the handoff process needs to be estimated in order to evaluate the system performance and to be used in system dimensioning. Although the signaling load will increase when the density of small cells increases, IRH shows insightful information regarding the signaling load that macro cells need to handle due to UEs' mobility. The macro cells will be involved in most of the handoffs in the system when the density of small cells is low. Furthermore, the signaling load handled by the macro cells and the signaling load handled by different small cells keep increasing when the density of small cells increases from low values to moderate values. However, the load handled by the macro cells starts decreasing when the density of small cells increases from moderate values to high values, despite the total signaling load in the system increasing when the density of small cells increases. This could be used as guidelines when considering expansion. Deploying high dense small cells in the network 
will not only enhance the system capacity and the data rate but also release more resources and minimize the signaling load at the macro cells.

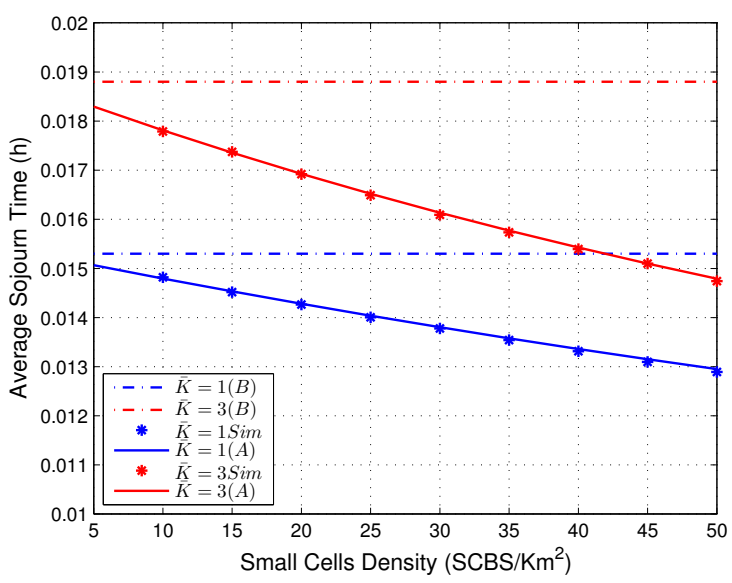

Fig. 11: Small cell sojourn time where $\lambda_{w}=0.01$.

The expectation of the small cell sojourn time when $\lambda_{w}=0.01$ and $\lambda_{w}=100$ (high value of $\lambda_{w}$ is more suitable for walking users [11]) is shown in Fig. 11 and Fig. 12. It is seen that the scenario (A) is very accurate in different densities of HetNets and the small cell sojourn is minimized in the dense HetNet due to the small cells overlapping. Furthermore, it is also seen that when the overlaps are ignored, e.g. scenario (B) [10], the small cell sojourn time becomes independent of the small cell density. However, our analysis (A) and the simulations show that the cell sojourn time is not only affected by the number of tiers and the small cells' footages, but also affected by the small cell density in the networks. The gap between the two scenarios (A) and (B) increases when the small cell density increases and/or the transmit power of the small cells increases. This implies that the analysis becomes inaccurate when ignoring the overlaps.

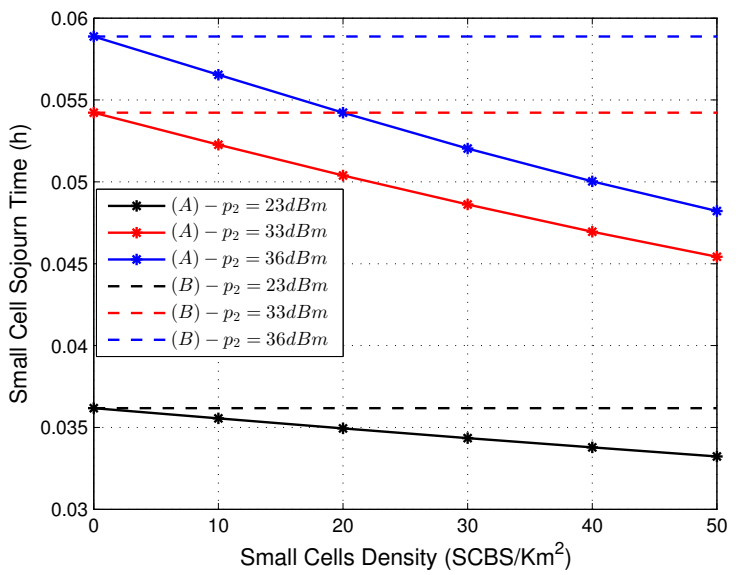

Fig. 12: Sojourn time where $v=1, \lambda_{w}=100, \bar{K}=1$ and $S=60 \mathrm{Sec}$.

Fig. 13 shows the macro cell sojourn time for different values of the mobility parameter $\lambda_{w}$. In this figure, two scenarios are considered. The first scenario shows the impact of the small cells with different densities on the macro cell sojourn time in multi-tier networks. The second scenario $(N o-S C s)$ represents the macro cell sojourn time when no small cells are deployed in the network [11] (single-tier networks). It can be seen that when the small cell density increases the macro cell sojourn time decreases. In the singletier network (e.g. No-SCs scenario), the macro cell sojourn time depends on the macro cell density and the total transition time, while the macro cell sojourn time in the HetNets is affected by the density and the footages of the small cells in addition to the macro cell density and the total transition time. Deploying more small cells will minimize the macro cell sojourn time as the probability of the UEs encountering the macro-small boundaries is greater than the probability of them encountering the macro-macro boundaries in a dense small cell network. The cell sojourn time which is shown in Figs 11,12 and 13, can also convey some conclusions regarding the resources utilization. For instance, the small cell sojourn time is minimized when the density of small cells increases. This means that the requested frequency resources by UEs from each small cell are minimized because the area served by each small cell is minimized and UEs will spend less time in each small cell individually when the density increases. The macro cell sojourn time is also minimized and the requested resources are also minimized when the density of small cells increases. This is reasonable since the coverage of small cells is maximized and the time that the UEs spend associating to the macro cells is minimized which helps to boost the system capacity and free more resources at the overloaded macro cells.

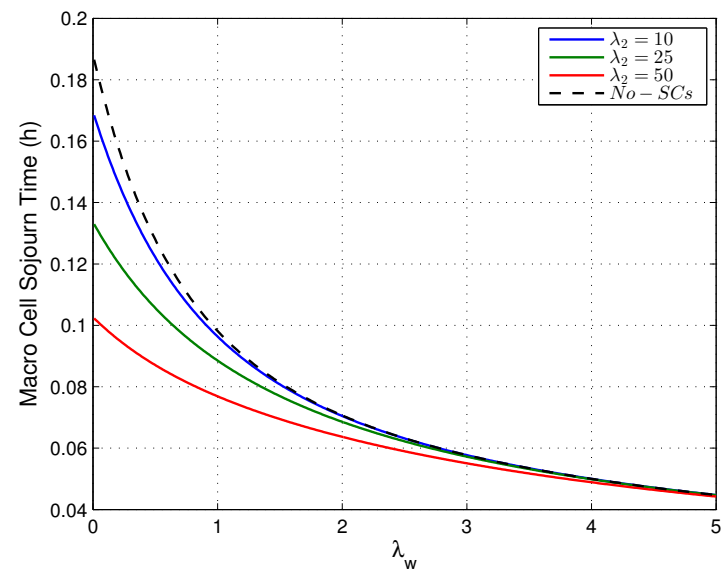

Fig. 13: Macro cell sojourn time, where $N o-S C s$ represents single-tier network [11].

\section{CONCLUSION AND REMARKS}

In this paper, the Stochastic Geometry tool was used to establish a mobility framework to model and analyse the main mobility parameters such as the handoff rate and the cell sojourn time as well as the expected time that the UEs spend in the coverage of the small cells. In the proposed framework, the overlaps among the small cells from different tiers on a reference UE's path was taken into consideration. The results showed that ignoring the overlaps can affect the accuracy of the cell sojourn time and handoff rate significantly. The IAH rate and the IRH rate were introduced to illustrate the load and the resources required at the different network tiers, especially at the overloaded macro cells. The simulation results showed that increasing the small cell density can reduce 
the load for the macro cells not only because the UEs will spend more time associating with the high frequency small cells but also more overlaps will take place among the high frequency small cells which minimize the fraction of handoffs managed by the macro cells. It was furthermore shown that the small cell sojourn time becomes independent of the small cell density when the overlaps are not considered. The results and analysis in the paper can be extended to study other aspects of the mobility management in the dense HetNet such as the ping pong and handoff failure rate, as well as to estimate the UE's speed. These results also have other applications such as estimating the potential offloading opportunity to the small cells in inter-frequency HetNets which is essential in the small cell discovery analysis to boost the system energy efficiency. Another application of the research work in this paper is to estimate the resource utilization in the system (e.g. frequency and signaling) which can help greatly in the system design and dimentioning.

\section{APPENDIX A}

Given that the $i$ th and the $(i+1)$ th small cells with coverage $C_{i}$ and $C_{i+1}$ respectively are overlapped on $\mathcal{P}_{0}$. From Fig. 4, the $i$ th small cell overlaps with the $(i+1)$ th small cell on $\mathcal{P}_{0}$ if $\bar{y}_{i}$ and $\bar{y}_{i+1}$ are at distance $\bar{C}_{i}$ or less. Thus, any overlap coverage can be expressed as:

$$
\mathfrak{C}_{i}= \begin{cases}\bar{C}_{i}-\left\|\bar{y}_{i+1}-\bar{y}_{i}\right\|, & \left\|\bar{y}_{i+1}-\bar{y}_{i}\right\|<\bar{C}_{i} \\ 0, & \text { otherwise }\end{cases}
$$

The PDF of $\bar{C}_{i}$ is the convolution of the PDFs of $C_{i}$ and $C_{i+1}$. Since $C_{i}$ and $C_{i+1}$ are independent random variables, the joint PDF of both $C_{i}$ and $C_{i+1}$ can be expressed as $f_{C_{i} \underline{C}_{i+1}}\left(c_{i}, c_{i+1}\right)=f_{C_{i}}\left(c_{i}\right) f_{C_{i+1}}\left(c_{i+1}\right)$. Therefore the PDF of $\bar{C}_{i}$ is obtained as:

$$
\begin{aligned}
f_{\bar{C}_{i}}(\bar{c}) & =\int_{-\infty}^{\infty}\left(\frac{d}{d \bar{c}} \int_{0}^{\bar{c}-c_{i}} f_{C_{i} C_{i+1}}\left(c_{i}, c_{i+1}\right) d c_{i+1}\right) d c_{i} \\
& =\int_{-\infty}^{\infty} f_{C_{i} C_{i+1}}\left(c_{i}, \bar{c}-c_{i}\right) d c_{i} \\
& \stackrel{(a)}{=} \int_{0}^{\bar{c}} \frac{\bar{c} c_{i}\left(\bar{c}-c_{i}\right) d c_{i}}{32 r_{i}^{2} r_{i+1}^{2} \sqrt{1-\frac{c_{i}^{2}}{4 r_{i}^{2}}} \sqrt{1-\frac{\left(\bar{c}-c_{i}\right)^{2}}{4 r_{i+1}^{2}}}} \\
& \stackrel{(b)}{=} \int_{0}^{\bar{c}} \frac{\bar{c} c_{i}\left(\bar{c}-c_{i}\right) d c_{i}}{16 r^{3} \sqrt{8 r^{2}-c_{i}^{2}-\left(\bar{c}-c_{i}\right)^{2}}}
\end{aligned}
$$

where (a) follows from Lemma 3 and from the fact that all small cells have the same distribution around $\mathcal{P}_{0}$, and (b) follows from $r_{i}=r, \forall i$. The expected value of $\bar{C}_{i}$ can be obtained as:

$$
\mathbb{E}\left[\bar{C}_{i}\right]=\int_{0}^{4 r} \bar{c} f_{\bar{C}_{i}}(\bar{c}) d \bar{c}
$$

where the integral limits follow from $C_{i}$ and $C_{i+1}$ being independent and from the fact that the maximum summation of both small cell coverages can be $2 r_{i}+2 r_{i+1}=4 r$ when both are maximum $C_{i}=2 r_{i}$ and $C_{i+1}=2 r_{i+1}$, and the minimum summation of both small cells coverages can be 0 when both are minimum $C_{i}=C_{i+1}=0$ as shown in Lemma 3 . Given that the $i$ th and $(i+1)$ th small cells overlapped on $\mathcal{P}_{0}$, the distance between $\bar{y}_{i}$ and $\bar{y}_{i+1}$ is uniformly distributed in the range $\left[0, \bar{C}_{i}\right]$. Therefore, the expectation of the one overlap coverage becomes:

$$
\mathbb{E}\left[\mathfrak{C}_{i}\right]= \begin{cases}\frac{\mathbb{E}\left[\bar{C}_{i}\right]}{2}, & \left\|\bar{y}_{i+1}-\bar{y}_{i}\right\|<\bar{C}_{i} \\ 0, & \text { otherwise }\end{cases}
$$

The results in Eq. (23) is reached after solving Eq. (A3).

\section{APPENDIX B}

Since the overlap between the $i$ th and the $(i+1)$ th small cells occurs when the distance between $\bar{y}_{i}$ and $\bar{y}_{i+1}$ is equal or less than $\bar{C}_{i}$, the number of overlaps is expressed as:

$$
N_{O L}=\sum_{i=2}^{N_{0}} \mathbf{1}\left(\left\|\bar{y}_{i}-\bar{y}_{i-1}\right\| \leq \bar{C}_{i-1}\right)
$$

the expected number of overlaps can be expressed as:

$$
\mathbb{E}\left[N_{O L}\right]=\mathbb{E}\left[N_{O L, \max }\right] \mathbb{P}_{O L}
$$

where $\mathbb{P}_{O L}$ is defined as the probability of two consecutive small cells with coverages $C_{i}$ and $C_{i+1}$ overlapping on $\mathcal{P}_{0}$. Given that the number of small cells in $\mathscr{A}_{2}$ is $N_{0}$, the maximum overlaps can take different values, for instance when $W_{1}$ is not located in the coverage of the small cells, the maximum number of overlaps occur on the path will be $N_{0}-1$. However, when $W_{1}$ is located in the coverage of the small cells, the maximum number of overlaps that can take place on $\mathcal{P}_{0}$ is either $N_{0}$ when $W_{1}$ is located in coverage of small cell that its SCBS is not located in $\mathscr{A}_{2}$ as shown in Fig. 2, or $N_{0}-1$ when $W_{0}$ is located in a small cell whose SCBS belongs to $\mathscr{A}_{2}$. Therefore, the expectation of the maximum number of overlaps that can occur on the path can be expressed as

$$
\mathbb{E}\left[N_{O L, \max }\right]=\mathcal{A}_{1}\left(\frac{\bar{\lambda}}{2 \sqrt{\lambda_{w}}}-1\right)+\frac{\mathcal{A}_{\bar{K}}}{2}\left(\frac{\bar{\lambda}}{\sqrt{\lambda_{w}}}-1\right)
$$

where $\frac{\bar{\lambda}}{2 \sqrt{\lambda_{w}}}$ represents the expected number of SCBSs located in the area $\mathscr{A}_{2}=2 r\left\|W_{0}-W_{1}\right\|$. Since the locations of the path points follow a PPP, the probability that the overlap occurs, is obtained from the null probability [15] as shown:

$$
\begin{aligned}
\mathbb{P}_{O L} & =1-\mathbb{P}[\text { No Overlap }]=1-\mathbb{P}\left[\left\|\bar{y}_{i+1}-\bar{y}_{i}\right\|>\mathbb{E}\left[\bar{C}_{i}\right]\right] \\
& =1-\exp \left(-\bar{\lambda} \int_{0}^{4 r} \bar{c} f_{\bar{C}_{i}}(\bar{c})\right)
\end{aligned}
$$

where $\exp \left(-\bar{\lambda} \int_{0}^{4 r} \bar{c} f_{\bar{C}_{i}}(\bar{c})\right)$ represents the probability of no overlap occurring or the probability that $\bar{y}_{i+1}$ is at distance greater than $\bar{C}_{i}$ from $\bar{y}_{i}$. The desired result in Eq. (24) is reached after substituting Eq. (B4) and Eq. (B3) in Eq. (B2).

\section{APPENDIX C}

Since the $i$ th small cell of the $m$ th overlaps with the $(i+1)$ th small cell of the $n$th tier on $\mathcal{P}_{0}$ when $\bar{z}_{i}$ and $\bar{z}_{i+1}$ are at distance $\bar{C}_{m n}$ or less, the overlap coverage between two small cells can be expressed similar to Eq. (A4) as:

$$
\mathbb{E}\left[\mathfrak{C}_{m n}\right]= \begin{cases}\frac{\mathbb{E}\left[\bar{C}_{m n}\right]}{2}, & \left\|\bar{z}_{i+1}-\bar{z}_{i}\right\|<\bar{C}_{m n} \\ 0, & \text { otherwise }\end{cases}
$$

The expectation of one coverage can be obtained as:

$$
\mathbb{E}\left[\bar{C}_{m n}\right]=\int_{0}^{2 r_{m}+2 r_{n}} \bar{c} f_{\bar{C}_{m n}}(\bar{c}) d \bar{c}
$$

where $f_{\bar{C}_{m n}}(\bar{c})$ is the PDF of $\bar{C}_{m n}$ is the convolution of the PDF of $C_{m}^{m n}$ and $C_{n}$ and similar to Eq. (A2) as:

$$
\begin{aligned}
f_{\bar{C}_{m n}}(\bar{c}) & =\int_{-\infty}^{\infty}\left(\frac{d}{d \bar{c}} \int_{0}^{\bar{c}-c_{m}} f_{C_{m} C_{n}}\left(c_{m}, c_{n}\right) d c_{n}\right) d c_{m} \\
& =\int_{0}^{\bar{c}} f_{C_{m} C_{n}}\left(c_{m}, \bar{c}-c_{m}\right) d c_{m} \\
& =\int_{0}^{\bar{c}} \frac{\bar{c} c_{m}\left(\bar{c}-c_{m}\right) d c_{m}}{16 r_{m}^{2} r_{n}^{2} \sqrt{8-\frac{c_{m}^{2}}{r_{m}^{2}}-\frac{\left(\bar{c}-c_{m}\right)^{2}}{r_{n}^{2}}}}
\end{aligned}
$$


Given that the $i$ th small cell is from the $m$ th tier, the $(i+$ 1)th small cell can be from any tier. The probability that the $(i+1)$ th small cell from the $n$th tier is $\frac{\bar{\lambda}_{n}}{\lambda_{\bar{K}}}$. Therefore, the expected value in Eq. (28) is reached after solving Eq. (C2).

\section{APPENDIX D}

Assume that $O$ point is located on $\mathcal{P}_{0}$ where the received power from both the $i$ th and $(i+1)$ th small cells are equal as shown in Fig. 4. Since the cell association among small cells is based on the maximum received power, the average received power at $O$ from both the $i$ th small cell and the $(i+1)$ th small cell can be expressed as:

$$
\frac{p_{m} \mathscr{L}_{2}}{\left(\sqrt{\tau_{i}^{2}+\Psi_{m, 1}^{2}}\right)^{\alpha_{m}}}=\frac{p_{n} \mathscr{L}_{2}}{\left(\sqrt{\tau_{i+1}^{2}+\Psi_{n, i+1}^{2}}\right)^{\alpha_{n}}}
$$

where $\Psi_{m, i}=\left\|\bar{z}_{i}-O\right\|$ and $\Psi_{n, i+1}=\left\|\bar{z}_{i+1}-O\right\|$ represent one side coverage served by the $i$ th small cell and the $(i+$ 1)th small cell on $\mathcal{P}_{0}$ respectively. Since the small cells from different tiers can be located at any distance from $\mathcal{P}_{0}$ and they have different transmit powers and path-loss exponents, the point $O$ can be located either between $z_{i}$ and $z_{i+1}$, before $z_{i}$ or after $z_{i+1}$. Without loss of generality, assuming that $\tau_{i}=$ $\tau_{i+1}=0$, the one side coverage of the $i$ th small cell of the $m$ th tier can be obtained as:

$$
\begin{aligned}
\Psi_{m, i} & =\left(\frac{p_{n}}{p_{m}}\right)^{\frac{-1}{\alpha_{m}}} \Psi_{n, i+1}^{\frac{\alpha_{n}}{\alpha_{m}}} \\
& \stackrel{(a)}{=} \frac{\left(\frac{p_{n}}{p_{m}}\right)^{\frac{-1}{\alpha_{m}}}}{1+\left(\frac{p_{n}}{p_{m}}\right)^{\frac{-1}{\alpha_{m}}}}\left\|\bar{z}_{i}-\bar{z}_{i+1}\right\|
\end{aligned}
$$

Note that $\Psi_{m, i}=r_{m}$ and $\Psi_{n, i+1}=r_{n}$ when both the $i$ th and the $(i+1)$ th do not overlap on $\mathcal{P}_{0}$. (a) follows from $\|$ $\bar{z}_{i}-\bar{z}_{i+1} \|=\Psi_{m, i}+\Psi_{n, i+1}$ and $\alpha_{m}=\alpha_{n}$. The footage of the $i$ th small cell and served by the $(i+1)$ th small cell can be obtained as:

$$
\begin{aligned}
\chi_{n \mapsto m} & =r_{m}-\Psi_{m, i} \\
& \stackrel{(b)}{=} r_{m}-\frac{\left(\frac{p_{n}}{p_{m}}\right)^{\frac{-1}{\alpha_{m}}}\left(r_{m}+r_{n}-\mathfrak{C}_{m n}\right)}{1+\left(\frac{p_{n}}{p_{m}}\right)^{\frac{-1}{\alpha_{m}}}} \\
& \stackrel{(c)}{=} \frac{\mathfrak{C}_{m n}\left(\frac{p_{n}}{p_{m}}\right)^{\frac{-1}{\alpha_{m}}}}{1+\left(\frac{p_{n}}{p_{m}}\right)^{\frac{-1}{\alpha_{m}}}}
\end{aligned}
$$

where (b) follows from Eq. (D2) and $\left\|\bar{z}_{i}-\bar{z}_{i+1}\right\|=r_{m}+$ $r_{n}-\mathfrak{C}_{m n}$, and (c) follows from $r_{m}=r_{n}\left(\frac{p_{n}}{p_{m}}\right)^{\frac{-1}{\alpha_{m}}}$. Given that the $i$ th small cell and $(i+1)$ th small cell overlap on $\mathcal{P}_{0}$, the expectation of $\mathfrak{C}_{m n}$ is obtained in Lemma 7. $\mathbb{E}\left[\chi_{n \mapsto m}\right]$ is expressed as in Eq. (32).

\section{APPENDIX E}

Given that the reference small cell crossed by $\mathcal{U}_{0}$, the sojourn time that $\mathcal{U}_{0}$ stays in the reference small cell when $V=v$ and $\bar{K}=1$ can be expressed as:

$$
\mathcal{S} \mathcal{T}_{i}^{0}=\frac{C_{i}-\Xi}{v}
$$

where $\Xi$ represents the footage of the reference small cell on the path and served by other small cells due to overlapping. $\Xi$ can take value between 0 when no overlap occurs, and $C_{i}$ when one overlap or more occur with other small cells on the path. Given that the $i$ th small cell has $C_{i}$ coverage on the path and overlaps with other small cells, the expectation of $\Xi$ is expressed as:

$$
\mathbb{E}[\Xi]=2 \mathbb{P}_{O L} \mathbb{E}[\chi]
$$

where $\mathbb{P}_{O L}$ is the probability that the $i$ th small cell overlaps with another small cell on $\mathcal{P}_{0}$ and is obtained in Eq. (B4), and $\mathbb{E}[\chi]$ is the expected value of the $i$ th small cell's footage on $\mathcal{P}_{0}$ served by the $(i+1)$ th small cell or the $(i-1)$ th small cell and is obtained in Eq. (32). When $\bar{K}>1$, the expectation of the $i$ th small cell's footage of $m$ th tier served by the small cell of the $n$th tier becomes $\mathbb{P}_{O L, m n} \sum_{n=1}^{\bar{K}} \frac{\bar{\lambda}_{n}}{\lambda_{\bar{K}}} \mathbb{E}\left[\chi_{n \mapsto m}\right]$. Since the $i$ th small cell of the $m$ th tier can overlap with more than one small cell, the expectation $\mathbb{E}[\Xi]$ is obtained as:

$$
\mathbb{E}[\Xi]=\left(\sum_{n=1}^{\bar{K}} \frac{\mathbb{P}_{O L, m n} \bar{\lambda}_{n}}{\bar{\lambda}_{\bar{K}}} \mathbb{E}\left[\chi_{n \mapsto m}\right]+\sum_{q=1}^{\bar{K}} \frac{\mathbb{P}_{O L, m q} \bar{\lambda}_{q}}{\bar{\lambda}_{\bar{K}}} \mathbb{E}\left[\chi_{q \mapsto m}\right]\right)
$$

Since the locations of SCBSs are uncorrelated and the expected value of the sojourn time in the reference small cell of the $m$ th tier can be expressed as:

$$
\mathbb{E}\left[S \mathcal{T}_{m}^{0}\right]=\frac{1}{v}\left(\mathbb{E}\left[C_{m}\right]-\mathbb{E}[\Xi]\right)
$$

There are $\bar{K}$ small cell tiers in the network, therefore, the expected value of the sojourn time in any small cell during one transition can be expressed as:

$$
\mathbb{E}\left[S \mathcal{S T}_{S C S}^{0}\right]=\sum_{m=1}^{\bar{K}} \frac{\mathbb{E}\left[\mathcal{S} \mathcal{T}_{m}^{0}\right] \bar{\lambda}_{m}}{\bar{\lambda}_{\bar{K}}}
$$

The result in Eq. (33) is reached.

\section{APPENDIX F}

Without loss of generality, assume that the destination waypoint is located at the origin and the distance to the small cell of interest of the $m$ th tier is donated by $\mathfrak{r}_{0}$. The probability of this point associated to the small cell of interest is a conditional probability:

$$
\begin{aligned}
& \mathbb{P}_{\mathcal{S} \mathcal{T}, m}=\mathbb{P}\left[\mathfrak{r}_{0}<r_{m} \mid \hat{\rho}_{m}>\max _{n \in[2,3, \cdots, K]} \rho_{n, 0}\right] \\
& =\mathbb{P}\left[\mathfrak{r}_{0}<\left(\frac{p_{\text {min }}}{\mathscr{L}_{2} p_{m}}\right)^{-\frac{1}{\alpha_{m}}}\right] \prod_{n=2}^{K} \mathbb{P}\left[\frac{p_{m} \mathscr{L}_{2}}{\mathfrak{r}_{0}^{\alpha_{m}}}>\frac{p_{n} \mathscr{L}_{2}}{R_{n, 0}^{\alpha_{n}}}\right] \\
& =\left(\frac{\pi\left(\frac{p_{m i n}}{\mathscr{L}_{2} p_{m}}\right)^{-\frac{2}{\alpha_{m}}}}{\mathscr{A}_{m}}\right)\left(\prod_{n=2}^{K} \mathbb{P}\left[\mathfrak{r}_{0}<\left(\frac{p_{m} R_{n, 0}^{\alpha_{n}}}{p_{n}}\right)^{\frac{1}{\alpha_{m}}}\right]\right) \\
& =\left(\frac{\pi\left(\frac{p_{m i n}}{\mathscr{L}_{2} p_{m}}\right)^{-\frac{2}{\alpha_{m}}}}{\mathscr{A}_{m}}\right)\left(\int _ { 0 } ^ { \infty } \prod _ { n = 2 } ^ { K } \operatorname { e x p } \left(-\pi \lambda_{n}\left(\frac{p_{n}}{p_{m}}\right)^{\frac{2}{\alpha_{n}}}\right.\right. \\
& \left.\left.R_{n, 0}^{\frac{2 \alpha_{m}}{\alpha_{n}}}\right) f_{R_{n, 0}}\left(R_{n, 0}\right)\right)
\end{aligned}
$$

where $\mathbb{P}\left[\mathfrak{r}_{0}<r_{m}\right]$ is the probability that the destination waypoint is in the footage of the small cell of interest, $\hat{\rho}_{m}$ and $\rho_{n, 0}$ are the received powers from the small cell of interest of $m$ th tier and the received power from the nearest small cell of $n$th tier respectively, and $\mathbb{P}\left[\hat{\rho}_{m}>\max _{n \in[2,3, \cdots, K]} \rho_{n, 0}\right]$ is the probability that $\mathcal{U}_{0}$ at the destination waypoint receives the maximum received power from the small cell of interest and $R_{n, 0}$ is the distance between the nearest small cell of the $n$th tier to the destination waypoint. Since the locations of both waypoints and SCBSs are uncorrelated and randomly 
distributed in the network, the variable $R_{n, 0}$ is assumed to have a Rayleigh distribution with PDF $2 \pi \lambda_{n} R_{n, 0} e^{-\pi \lambda_{n} R_{n, 0}^{2}}$. Eq. (F1) becomes:

$$
\begin{array}{r}
\mathbb{P}_{\mathcal{S}, m=}=\left(\frac{\pi\left(\frac{p_{\text {min }}}{\mathscr{L}_{2} p_{m}}\right)^{-\frac{2}{\alpha_{m}}}}{\mathscr{A}_{m}}\right)\left(\int _ { 0 } ^ { \infty } \prod _ { n = 2 } ^ { K } \operatorname { e x p } \left(-\pi \lambda_{n}\left(\frac{p_{n}}{p_{m}}\right)^{\frac{2}{\alpha_{n}}}\right.\right. \\
\left.\left.R_{n, 0}^{\frac{2 \alpha_{m}}{\alpha_{n}}}\right) 2 \pi \lambda_{n} R_{n, 0} e^{-\pi \lambda_{n} R_{n, 0}^{2}} d R_{n, 0}\right) \\
=\left(\frac{\pi\left(\frac{p_{\text {min }}}{\mathscr{L}_{2} p_{m}}\right)^{-\frac{2}{\alpha_{m}}}}{\mathscr{A}_{m}}\right)\left(2 \pi \prod _ { n = 2 } ^ { K } \lambda _ { n } \int _ { 0 } ^ { \infty } R _ { n , 0 } \operatorname { e x p } \left(-\pi \lambda_{n}\right.\right. \\
\left.\left.\left(\left(\frac{p_{n}}{p_{m}}\right)^{\frac{2}{\alpha_{n}}} R_{n, 0}^{\frac{2 \alpha_{m}}{\alpha_{n}}}+R_{n, 0}^{2}\right)\right) d R_{n, 0}\right)
\end{array}
$$

when $\alpha_{m}=\alpha_{n}, \mathbb{P}_{\mathcal{S T}, m}$ is obtained after solving Eq. (F2).

\section{REFERENCES}

[1] H. Zhu and J. Wang, "Chunk-Based Resource Allocation in OFDMA Systems - Part I: Chunk Allocation," IEEE Transactions on Communications, vol. 57, pp. 2734 - 2744, Sep. 2009.

[2] H. Zhu and J. Wang, "Chunk-Based Resource Allocation in OFDMA Systems - Part II: Joint Chunk, Power and Bit Allocation," IEEE Transactions on Communications, vol. 60, pp. 499 - 509, Feb. 2012.

[3] I. F. Akyildiz, J. S. M. Ho, and W. Wang, "Mobility Management in Next-Generation Wireless Systems," Proc. IEEE, vol. 87, pp. 1347 1384, Aug. 1999.

[4] A. Merwaday and I. Gven, "Handover Count Based Velocity Estimation and Mobility State Detection in Dense HetNets," IEEE Transactions on Wireless Communications, vol. 15, pp. 4673-4688, Jul. 2016.

[5] A. Merwaday, I. Gven, W. Saad, A. Mehbodniya, and F. Adachi, "Sojourn Time-Based Velocity Estimation in Small Cell Poisson Networks," IEEE Communication Letters, vol. 20, pp. 340 - 341, Feb. 2016.

[6] D. Lpez-Prez, I. Gvenc, and X. Chu, "Mobility Management Challenges in 3GPP Heterogeneous Networks," IEEE Communication Magazine, vol. 50, pp. 70-78, Dec. 2012.

[7] O. Onireti, A. Imran, M. A. Imran, and R. Tafazolli, "Energy efficient inter-frequency small cell discovery in heterogeneous networks," IEEE Transactions on Vehicular Technology, vol. 65, pp. 7122-7135, Sept 2016.

[8] A. Prasad, O. Tirkkonen, P. Lunden, O. N. Yilmaz, L. Dalsgaard, and C. Wijting, "Energy-Efficient Inter-Frequency Small Cell Discovery Techniques for LTE-Advanced Heterogeneous Network Deployments," IEEE Communications Magazine, vol. 51, pp. 72-81, May 2013.

[9] A. Mahbas, H. Zhu, and J. Wang, "The optimum rate of inter-frequency scan in inter-frequency hetnets," in 2017 IEEE International Conference on Communications (ICC), pp. 1-6, May 2017.

[10] S. Shin, U. Lee, F. Dressler, and H. Yoon, "Analysis of Cell Sojourn Time in Heterogeneous Networks With Small Cells," IEEE Communication Letters, vol. 20, pp. 788 - 791, Apr. 2016.

[11] X. Lin, R. K. Ganti, P. J. Fleming, , and J. G. Andrews, "Towards Understanding the Fundamentals of Mobility in Cellular Networks," IEEE Transactions on Wireless Communications, vol. 12, pp. 16861698, Apr. 2013.

[12] Y. Hong, X. Xu, M. Tao, J. Li, and T. Svensson, "Cross-tier Handover Analyses in Small Cell Networks: A Stochastic Geometry Approach," IEEE International Conference on Communication (ICC), pp. 34293434, Jun. 2015.

[13] W. Bao and B. Liang, "Stochastic Geometric Analysis of User Mobility in Heterogeneous Wireless Networks," IEEE Journal on Selected Areas in Communications, vol. 33, pp. 2212 - 2225, Oct. 2015.

[14] T. S. Rappaport, S. Sun, R. Mayzus, H. Zhao, Y. Azar, K. Wang, G. N Wong, J. K. Schulz, MathewSamimi, and F. Gutierrez, "Millimeter Wave Mobile Communications for 5G Cellular: It Will Work!," IEEE Access, vol. 1, pp. 335-349, 2013.

[15] D. Stoyan, W. S. Kendall, and J. Mecke, Stochastic Geometry and Its Applications. John Wiley and Sons Ltd., 1995.

[16] A. Mahbas, H. Zhu, and J. Wang, "Unsynchronized Small Cells with a Dynamic TDD System in a Two-Tier HetNet," IEEE 83rd Vehicular Technology Conference: VTC2016-Spring, pp. 1-6, 2016.
[17] C. Bettstetter, H. Hartenstein, and X. Perez-Costa, "Stochastic Properties of the Random Waypoint Mobility Model," Wireless Networks, vol. 10, p. 555567, Sep. 2004

[18] J. Broch, D. A. Maltz, D. B. Johnson, Y.-C. Hu, and J. Jetcheva, "A Performance Comparison of Multi-Hop Wireless Ad Hoc Network Routing Protocols," ACM International Conference on Mobile Comput. and Networking, Oct. 1998.

[19] M. M. Zonoozi and P. Dassanayake, "User Mobility Modeling and Characterization of Mobility Patterns," IEEE Journal on Selected Areas in Communications, vol. 15, pp. 1239 - 1252, Sep. 1997.

[20] M. Haenggi, Stochastic Geometry for Wireless Networks. Cambridge University Press, 2012.

[21] C. Bettstetter, G. Resta, and P. Santi, "The node distribution of the random waypoint mobility model for wireless ad hoc networks," IEEE Transactions on Mobile Computing, vol. 2, pp. 257-269, July 2003.

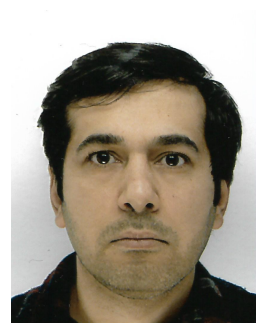

Ali J. Mahbas received the B.S. degree in Electronic and Communication Engineering, the M.S. degree in Communication Network Planning and Management and the Ph.D. degree in Electronic Engineering from University of Technology, Iraq, University of Portsmouth, UK, University of Kent, UK, in 2005, 2009 and 2017, respectively.

Dr. Mahbas has more than 10 years experience in the area of wireless communications.

$\mathrm{He}$ is currently working for the Iraqi Ministry of Communications (MOC) and his research interests are in the areas of 5G, small cells, heterogeneous networks, mobility management, stochastic geometry modeling for cellular networks and device-todevice communications.

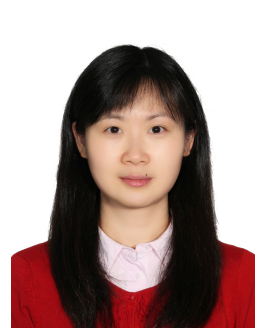

Huiling Zhu (SM'17) received the B.S degree from Xidian Univeristy, Xian, China, and the Ph.D. degree from Tsinghua University, Beijing, China. She is currently a Reader (Associate Professor) in the School of Engineering and Digital Arts, University of Kent, Canterbury, United Kingdom. Her research interests are in the area of wireless communications, covering topics such as radio resource management, MIMO, cooperative communications, deviceto-device communications, Cloud RAN, and small cells and heterogeneous networks. She received the best paper award from IEEE Globecom2011. She has participated in a number of European and industrial projects in these topics and was holding European Commission Marie Curie Fellowship from 2014 to 2016. She has served as the Publication Chair for IEEE WCNC 2013, Operation Chair for IEEE ICC 2015, Symposium Co-Chair for IEEE Globecom 2015 and IEEE ICC 2018, and Track Co-Chair of IEEE VTC2016-Spring and VTC2018-Spring. Currently, she serves as an Editor for IEEE Transactions on Vehicular Technology.

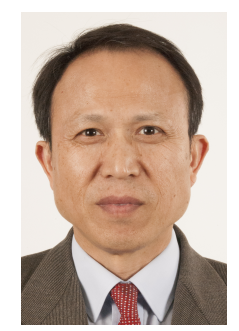

Jiangzhou Wang (F'17) is currently a Professor and the former Head of the School of Engineering and Digital Arts at the University of Kent, UK. He has published over 300 papers in international journals and conferences and four books in the area of wireless mobile communications.

Professor Wang is a Fellow of the Royal Academy of Engineering (UK) and Fellow of IEEE. He received the Best Paper Award from IEEE GLOBECOM2012 and was an IEEE Distinguished Lecturer from 2013 to 2014. He is the Technical Program Chair of IEEE ICC2019, Shanghai. He was the Executive Chair of IEEE ICC2015 London, and the TPC Chair of IEEE WCNC2013. He has served as an Editor for a number of international journals including IEEE Transactions on Communications from 1998 to 2013. 